\title{
COMPLEX MONGE-AMPÈRE EQUATION FOR MEASURES SUPPORTED ON REAL SUBMANIFOLDS
}

\author{
DUC-VIET VU
}

\begin{abstract}
Let $(X, \omega)$ be a compact $n$-dimensional Kähler manifold on which the integral of $\omega^{n}$ is 1 . Let $K$ be an immersed real $\mathcal{C}^{3}$ submanifold of $X$ such that the tangent space at any point of $K$ is not contained in any complex hyperplane of the (real) tangent space at that point of $X$. Let $\mu$ be a probability measure compactly supported on $K$ with $L^{p}$ density for some $p>1$. We prove that the complex Monge-Ampère equation $\left(d d^{c} \varphi+\omega\right)^{n}=\mu$ has a Hölder continuous solution.
\end{abstract}

Keywords: Monge-Ampère equation, generic CR submanifold.

\section{CONTENTS}

1. Introduction

2. Interpolation theory

3. Analytic discs partly attached to a generic submanifold

4. Some estimates for p.s.h. functions

5. Hölder continuity for super-potentials

References

\section{INTRODUCTION}

Let $X$ be a compact Kähler manifold of dimension $n$ and let $\omega$ be a fixed Kähler form on $X$ so normalized that $\int_{X} \omega^{n}=1$. The aim of this paper is to give a useful explicit class of measures for which the complex Monge-Ampère equation has a Hölder continuous solution. Recall that a real $\mathcal{C}^{1}$ manifold $K$ is said to be immersed in $X$ if there is an injective $\mathcal{C}^{1}$ immersion from $K$ to $X$. In this case we say that $K$ is an immersed $\mathcal{C}^{1}$ submanifold of $X$. An immersed real $\mathcal{C}^{1}$ submanifold $K$ of $X$ is said to be generic $C R$ (or generic for simplicity) in the sense of the Cauchy-Riemann geometry if the tangent space at any point of $K$ is not contained in a complex hyperplane of the tangent space at that point of $X$. Such a submanifold has the real dimension at least $n$. A function $\varphi: X \rightarrow[-\infty, \infty)$ is quasi-p.s.h. if it is locally the sum of a p.s.h. function and a smooth one. A quasi-p.s.h. function is said to be $\omega$-p.s.h. if we have $d d^{c} \varphi+\omega \geq 0$ in the sense of currents. The following is our main result.

Theorem 1.1. Let $K$ be a generic immersed $\mathcal{C}^{3}$ submanifold of $X$ of real codimension $d>0$. Let $\mu$ be a probability measure compactly supported on $K$ with $L^{p}$ density for some $p>1$.

Date: September 17, 2018.

This research is supported by grants from Région Ile-de-France. 
Then the Monge-Ampère equation $\left(d d^{c} \varphi+\omega\right)^{n}=\mu$ has an $\omega$-p.s.h. solution $\varphi$ which is Hölder continuous with Hölder exponent $\alpha$, for any positive number $\alpha<\frac{2(p-1)}{3 d(n+1) p}$.

Note that our proof still holds if $K$ is $\mathcal{C}^{2, \beta}$ for some $\beta \in(0,1)$. In this case one just needs to replace the $\mathcal{C}^{2,1 / 2}$ regularity in Section 3 by $\mathcal{C}^{2, \beta^{\prime}}$ one for $\beta^{\prime} \in(0, \beta)$. For simplicity, we only consider the $\mathcal{C}^{3}$ regularity as in Theorem 1.1. Secondly, if the Monge-Ampère equation has a Hölder continuous solution, then that solution is unique up to an additive constant. This is a direct consequence of results in [13, 6].

For a probability measure $\mu$ on $X$, the associated complex Monge-Ampère equation

$$
\left(d d^{c} \varphi+\omega\right)^{n}=\mu
$$

has been extensively studied since the fundamental paper [25] of Yau in which he proved that (1.1) has a unique smooth solution if $\mu$ is a (smooth) Riemannian volume form $v l_{X}$ of $X$. Later Kołodziej showed that the Monge-Ampère equation admits a unique continuous solution for a larger class of measures $\mu$ which contains $\mu=\operatorname{fvol}_{X}$ with $f \in L^{p}(X)$ for $p>1$, see [14, 13]. For the last measures, he also obtained Hölder regularity of the solution in [15]. The Hölder exponent of that solution is then made precise by Demailly, Dinew, Guedj, Hiep, Kolodziej and Zeriahi in [5] using the regularization method in [4] and the stability theorem in [7]. Moreover, in [11] Hiep obtains the Hölder regularity for $\mu=f v^{\prime} l_{Y}$, where $v l_{Y}$ is the volume form of a compact real hypersurface $Y$ of $X$ and $f \in L^{p}(Y)$ for $p>1$.

Recently, Dinh and Nguyên in [8] show that the class of probability measures $\mu$, for which (1.1) admits a Hölder continuous solution, is exactly the class of probability measures whose super-potentials are Hölder continuous, see Definition 1.3 below. They then recover the aforementioned results in [15, 11, 5]. By [8], we know that if a probability measure $\mu$ having a Hölder continuous super-potential of order $\beta \in(0,1]$, then the solution of (1.1) is Hölder continuous of order $\beta^{\prime}$ for any $0<\beta^{\prime}<2 \beta /(n+1)$. For more information on the complex Monge-Ampère equation, the readers may consult the survey [20].

Theorem 1.1] above combined with [8, Pro. 4.4] yields the following nice exponential estimate, see also [22, 9, 12].

Corollary 1.2. Let $K$ be a generic immersed $\mathcal{C}^{3}$ submanifold of $X$. Let $\tilde{K}$ be a compact subset of $K$. Then the restriction of the Lebesgue measure on $K$ to $\tilde{K}$ is moderate, that is, there exist two positive constants $\alpha$ and $c$ such that for any $\omega$-p.s.h. function $\varphi$ on $X$ with $\sup _{X} \varphi=0$ we have

$$
\int_{\tilde{K}} e^{-\alpha \varphi} d \operatorname{vol}_{K} \leq c
$$

Before presenting the idea of the proof of Theorem 1.1, we need to recall some definitions. Let $\mu$ be a probability measure on $X$. Let $\mathscr{C}$ be the set of $\omega$-p.s.h. functions $\varphi$ on $X$ such that $\int_{X} \varphi \omega^{n}=0$. We define the distance $\operatorname{dist}_{L^{1}}$ on $\mathscr{C}$ by putting

$$
\operatorname{dist}_{L^{1}}\left(\varphi_{1}, \varphi_{2}\right):=\int_{X}\left|\varphi_{1}-\varphi_{2}\right| \omega^{n},
$$

for every $\varphi_{1}, \varphi_{2} \in \mathscr{C}$. 
Definition 1.3. The super-potential of $\mu$ (of mean 0) is the function $\mathscr{U}: \mathscr{C} \rightarrow \mathbb{R}$ given by $\mathscr{U}(\varphi):=\int_{X} \varphi d \mu$. We say that $\mathscr{U}$ is Hölder continuous with Hölder exponent $\alpha \in(0,1]$ if it is so with respect to the distance dist $_{L^{1}}$.

By [8, The. 1.3, Cor. 4.5], Theorem 1.1 is a direct consequence of the following result.

Theorem 1.4. Let $K$ be a generic immersed $\mathcal{C}^{3}$ submanifold of $X$ of real codimension $d>0$. Let $\tilde{K}$ be a compact subset of $K$ and $\mathbf{1}_{\tilde{K}}$ the characteristic function of $\tilde{K}$. Let $v l_{K}$ be an arbitrary $\mathcal{C}^{3}$ Riemannian volume form of $K$. Then the super-potential of $\mathbf{1}_{\tilde{K}} v o l_{K}$ is Hölder continuous with Hölder exponent $\alpha$ for any positive number $\alpha<1 /(3 d)$.

Let $\mathbb{D}$ be the unit disc in $\mathbb{C}$ and let $\partial \mathbb{D}$ be the boundary of $\mathbb{D}$. A $\mathcal{C}^{1}$ analytic disc in $X$ is a $\mathcal{C}^{1}$ map from $\overline{\mathbb{D}}$ to $X$ which is holomorphic on $\mathbb{D}$. For a nonempty arc $I \subset \partial \mathbb{D}$, an analytic disc $f$ is said to be $I$-attached to a subset $K$ of $X$ if $f(I)$ belongs to $K$. When we do not want to mention $I$, we simply say an analytic disc partly attached to $K$. Throughout this paper, for every parameter $\tau$, we will systematically use the notation $\lesssim_{\tau}$ or $\lesssim$ which means $\leq$ up to a constant depending only on $(\tau, X, K, \omega)$ or on $(X, K, \omega)$ respectively. A similar convention is applied to $\gtrsim_{\tau}$ and $\gtrsim$.

The idea of the proof of Theorem 1.4 is as follows. Observe that the codimension $d$ of $K$ is at most equal to $n$. We consider below the case where $d=n$. The other cases can be deduced from it. Let $\varphi_{1}, \varphi_{2} \in \mathscr{C}$ and $\varphi:=\varphi_{1}-\varphi_{2}$. To show the Hölder regularity of the super-potential of $\mathrm{vol}_{K}$, by definition we need to bound the $L^{1}$-norm of $\varphi$ with respect to $\mathrm{vol}_{K}$ by a power of the $L^{1}$-norm of $\varphi$ on $X$. Since one can approximate any $\omega$-p.s.h. function on $X$ by a decreasing smooth ones (see [3]), it is enough to prove the desired property for smooth $\varphi_{1}, \varphi_{2}$ with $\varphi_{1} \geq \varphi_{2}$, see Proposition 5.1 and Lemma 5.2. In this case, $\varphi$ is smooth and nonnegative. This reduction is crucial in our proof. Observe that by compactness of $\tilde{K}$, it suffices to estimate

$$
\int_{\tilde{K}^{\prime}} \varphi d v o l_{K}
$$

for small open subsets $\tilde{K}^{\prime}$ of $\tilde{K}$. For each point $a \in K$, we will construct a $\mathcal{C}^{2,1 / 2}$ differentiable family $\tilde{F}_{\{\tau \in Z\}}$ of analytic discs partly attached to $K$ parameterized by $\tau$ in a compact manifold $Z$ of real dimension $(2 n-2)$ which roughly satisfies the following two properties:

(i) the restriction of $\tilde{F}$ to $\partial \mathbb{D} \times Z$ is a submersion onto an open neighborhood $K^{\prime}$ of $a$ in $K$, where we consider $\tilde{F}$ as a map from $\overline{\mathbb{D}} \times Z$ to $X$.

(ii) the restriction of $\tilde{F}$ to $\mathbb{D} \times Z$ is a diffeomorphism onto an open subset of $X$.

Put $\tilde{K}^{\prime}:=\tilde{K} \cap K^{\prime}$ for $a \in \tilde{K}$. These $\tilde{K}^{\prime}$ covers $\tilde{K}$. By the change of variables theorem and Property $(i)$, we have

$$
\int_{\tilde{K}^{\prime}} \varphi d v o l_{K} \leq \int_{K^{\prime}} \varphi d v o l_{K} \lesssim \int_{\partial \mathbb{D} \times Z} \varphi \circ \tilde{F}
$$

Since $\tilde{F}$ is holomorphic on $\mathbb{D}$ and $\mathcal{C}^{2}$ on $\overline{\mathbb{D}}$, observe that $\varphi \circ \tilde{F}$ is the difference of two $\mathcal{C}^{2}$ subharmonic functions on $\overline{\mathbb{D}}$.

Our second step is to bound $\int_{\partial \mathbb{D} \times Z} \varphi \circ \tilde{F}$ by a quantity involving $\int_{\mathbb{D} \times Z} \varphi \circ \tilde{F}$. For this purpose, we will establish a crucial inequality in dimension one which shows that $L^{1}$ norm on $\partial \mathbb{D}$ of a nonnegative $\mathcal{C}^{2}$ function on $\overline{\mathbb{D}}$ is bounded by a function of its $L^{1}$-norm on $\mathbb{D}$ and some Hölder norm of its Laplacian on $\mathbb{D}$. The ingredients for the proof of the last 
inequality are Riesz's representation formula and a general interpolation inequality for currents on manifolds with boundary. Note that a version of that interpolation inequality for manifolds without boundary was firstly used by Dinh and Sibony in [10].

The problem will be solved if one is able to bound $\int_{\mathbb{D} \times Z} \varphi \circ \tilde{F}$ by a constant times $\|\varphi\|_{L^{1}(X)}$. Taking into account Property $(i i)$, one is tempted to use the change of variables by $\tilde{F}$. However, the Jacobian of $\tilde{F}$ is small near the boundary $\partial \mathbb{D} \times Z$. This is due to a general fact that any family of analytic discs satisfying Property $(i)$ should degenerate at $\partial \mathbb{D}$ because of its attachment to $K$. So we need a precise control of the Jacobian of $\tilde{F}$ from below and prove some estimates on the integrals of p.s.h. functions and their $d d^{c}$ on a tubular neighborhood of $\tilde{K}$. These estimates are of independent interest. Consequently, we will get

$$
\int_{\mathbb{D} \times Z} \varphi \circ \tilde{F} \lesssim_{\alpha_{2}}\left(\int_{X} \varphi d v o l_{X}\right)^{\alpha_{2}},
$$

for any $\alpha_{2} \in(0,1 / n)$. Combining these above inequalities gives the Hölder regularity of the super-potential of $\mathrm{vol}_{K}$.

The paper is organized as follows. Section 2 is devoted to proving the above mentioned interpolation inequality for currents. In Section 3 , we construct the desired family of analytic discs $\tilde{F}$. In Section 4 , we present (1.2) and (1.3). Finally, we prove Theorem 1.4 in Section 5, At the beginning of Section 3, we will fix some notations which will be used for the rest of the paper.

Acknowledgement. The author would like to thank Tien-Cuong Dinh for introducing him this research topic and for his illuminating discussions. He also wants to express his gratitude to Lucas Kaufmann for fruitful discussions.

\section{INTERPOLATION THEORY}

Let $M$ be a compact smooth manifold of dimension $m$. Fix a partition of unity subordinated to a finite covering of local charts of $M$. For $k \in \mathbb{N}$ and $\alpha \in(0,1]$, let $\mathcal{C}^{k, \alpha}(M)$ be the space of $\mathcal{C}^{k}$ functions on $M$ whose partial derivatives of order $k$ are Hölder continuous of order $\alpha$. We endow the last space with the usual norm. For $t \in[0, \infty)$, denote by $\mathcal{C}^{t}(M)$ the space $\mathcal{C}^{[t], t-[t]}(M)$ where $[t]$ is the integer part of $t$. Let $\Lambda^{l} T^{*} M$ be the $l^{t h}$-exterior power of the cotangent vector bundle $T^{*} M$ for $1 \leq l \leq m$. Let $\mathcal{C}^{t}\left(M, \Lambda^{l} T^{*} M\right)$ be the set of $l$-differential forms with $\mathcal{C}^{l}$ coefficients. Using the above fixed partition of unity, we can equip $\mathcal{C}^{t}\left(M, \Lambda^{l} T^{*} M\right)$ with the norm $\|\cdot\|_{\mathcal{C}^{t}}$ which is the maximum of the $\mathcal{C}^{t}$ norms of its coefficients.

Let $T$ be an $l$-current of order 0 , i.e., there is a constant $C$ such that $|\langle T, \Phi\rangle| \leq C\|\Phi\|_{\mathcal{C}^{0}}$ for every smooth $(m-l)$-form $\Phi$. For $t \in[0, \infty)$, define

$$
\|T\|_{\mathcal{C}^{-t}}:=\sup _{\Phi \text { smooth },\|\Phi\|_{\mathcal{C}^{t}=1}}|\langle T, \Phi\rangle| .
$$

We will write $\|T\|$ instead of $\|T\|_{\mathcal{C}^{-0}}$ which is the usual mass norm of $T$. Dinh and Sibony in [10] proved that for any $t_{1}, t_{2} \in(0, \infty)$ with $t_{1}<t_{2}$, we have

$$
\|T\|_{\mathcal{C}^{-t_{2}}} \leq\|T\|_{\mathcal{C}^{-t_{1}}} \leq c\|T\|^{1-t_{1} / t_{2}}\|T\|_{\mathcal{C}^{-t_{2}}}^{t_{1} / t_{2}}
$$

for some constant $c$ independent of $T$. This inequality is very useful when dealing with continuous functionals on differential forms because one can reduce the problem to the 
smooth case. In this section, we will establish a generalization of (2.2) for compact smooth manifolds with boundary.

Let $M$ be a compact smooth manifold of dimension $m$ with boundary. Cover $M$ by a finite number of local charts $U_{j}$. Take a partition of unity $\phi_{j}$ subordinated to this covering. By the aid of these $\phi_{j}$, as above we can define the Banach spaces $\mathcal{C}^{t}(M)$ with the usual norms for $t \in[0, \infty)$. Denote by $\operatorname{Int} M$ the interior of $M$. Let $\mathcal{C}_{c}^{t}(\operatorname{Int} M)$ be the subspace of $\mathcal{C}^{t}(M)$ of $f \in \mathcal{C}^{t}(M)$ with compact support in $\operatorname{Int} M$. Let $\tilde{\mathcal{C}}^{t}(M)$ be the subspace of $\mathcal{C}^{t}(M)$ consisting of $f$ with $\left.f\right|_{\partial M} \equiv 0$. We can also define $\tilde{\mathcal{C}}^{t}\left(M, \Lambda^{l} T^{*} M\right)$ and $\mathcal{C}_{c}^{t}\left(M, \Lambda^{l} T^{*} M\right)$ in the same way as above.

Let $T$ be an $l$-current of order 0 on $\operatorname{Int} M$. Assume that its mass is finite, that is,

$$
\|T\|:=\sup _{\Phi \text { smooth },\|\Phi\|_{\mathcal{C}_{C}^{0}(\operatorname{Int} M)}=1}|\langle T, \Phi\rangle|<\infty .
$$

In our application, $M$ will be $\overline{\mathbb{D}}$ and $T$ will be the restriction of a continuous form on $\mathbb{C}$ to $\mathbb{D}$. By Riesz's representation theorem, $T$ is a differential form whose coefficients are Radon measures on $M$ with finite total variations. Hence, for any continuous differential form $\Phi$ on $\operatorname{Int} M$ with $\|\Phi\|_{\mathcal{C}^{0}}<\infty$, the value of $T$ at $\Phi$ is well-defined. Then the current $T$ can be extended to be a continuous linear functional on $\tilde{\mathcal{C}}^{t}\left(M, \Lambda^{l} T^{*} M\right)$. Let $\|T\|_{\tilde{\mathcal{C}}^{-t}(M)}$ be the norm of $T$ as a continuous linear functional on $\tilde{\mathcal{C}}^{t}\left(M, \Lambda^{l} T^{*} M\right)$. As mentioned at the beginning of the section, we will prove the following analogue of (2.2).

Proposition 2.1. Let $T$ be a l-current of order 0 on Int $M$. Assume that $T$ has finite mass. Let $t_{0}, t_{1}, t_{2} \in[0, \infty)$ with $t_{0}<t_{1}<t_{2}$. Let $t_{*}$ be the unique real number for which $t_{1}=$ $t_{*} t_{0}+\left(1-t_{*}\right) t_{2}$. Then we have

$$
\|T\|_{\tilde{\mathcal{C}}^{-t_{2}(M)}} \leq\|T\|_{\tilde{\mathcal{C}}^{-t_{1}(M)}} \leq C\|T\|_{\tilde{\mathcal{C}}^{-t_{0}(M)}}^{t_{*}}\|T\|_{\tilde{\mathcal{C}}^{-t_{2}(M)}}^{1-t_{*}},
$$

for some constant $C$ independent of $T$.

The remaining part of this section is devoted to prove the last proposition. Using a partition of unity as above, that proposition is a direct consequence of Corollary 2.9 at the end of this section. We first recall some notations and results from the interpolation theory of Banach spaces and refer to [17, 23] for a general treatment of the theory. Then we compute some interpolation spaces of $\tilde{\mathcal{C}}^{t}(M)$, see Corollary 2.8 below.

Let $A_{0}$ and $A_{1}$ be two Banach spaces which are continuously embedded to a Hausdorff topological vector space $\mathcal{A}$. Let $B_{0}$ and $B_{1}$ be two Banach spaces which are continuously embedded to a Hausdorff topological vector space $\mathcal{B}$. Let $T$ be a linear operator from $\mathcal{A}$ to $\mathcal{B}$. Assume that $\left.T\right|_{A_{j}}: A_{j} \rightarrow B_{j}$ are bounded for $j=0,1$. The interpolation theory of Banach spaces is to search for Banach subspaces $A \subset \mathcal{A}$ and $B \subset \mathcal{B}$ such that the restriction $\left.T\right|_{A}: A \rightarrow B$ is a bounded linear operator. The spaces $A$ and $B$ are called interpolation spaces. We will recall below a classical construction of such spaces.

For $0<t<\infty$ and $a \in A_{0}+A_{1}$, define

$$
K\left(t, a ; A_{0}, A_{1}\right):=\inf _{a=a_{0}+a_{1}}\left(\left\|a_{0}\right\|_{A_{0}}+t\left\|a_{1}\right\|_{A_{1}}\right),
$$

where $a_{0} \in A_{0}, a_{1} \in A_{1}$. Let $\alpha$ be a constant in $(0,1)$. The following class of Banach spaces is of great importance in the interpolation theory. 
Definition 2.2. Let $\left(A_{0}, A_{1}\right)_{\alpha, \infty}$ be the subspace of $A_{0}+A_{1}$ consisting of $a \in A_{0}+A_{1}$ for which the following quantity

$$
\|a\|_{\left(A_{0}, A_{1}\right)_{\alpha, \infty}}:=\sup _{t>0} t^{-\alpha} K\left(t, a ; A_{0}, A_{1}\right)
$$

is finite. The last formula defines a norm on $\left(A_{0}, A_{1}\right)_{\alpha, \infty}$ which make it to be a Banach space.

The following fundamental theorem explains the role of the space $\left(A_{0}, A_{1}\right)_{\alpha, \infty}$.

Theorem 2.3. [17, Th. 1.1.6] Let $A_{0}, A_{1}, B_{0}, B_{1}$ and $T$ be as above. Let $\alpha \in(0,1)$. Then the restriction $T_{\left(A_{0}, A_{1}\right)_{\alpha, \infty}}$ of $T$ to $\left(A_{0}, A_{1}\right)_{\alpha, \infty}$ is a bounded linear operator from $\left(A_{0}, A_{1}\right)_{\alpha, \infty}$ to $\left(B_{0}, B_{1}\right)_{\alpha, \infty}$ and

$$
\left\|\left.T\right|_{\left(A_{0}, A_{1}\right)_{\alpha, \infty}}\right\| \leq\left\|\left.T\right|_{A_{0}}\right\|^{1-\alpha}\left\|\left.T\right|_{A_{0}}\right\|^{\alpha},
$$

where $\|\cdot\|$ is the norm of bounded linear operators.

Let $m \in \mathbb{N}^{*}$ and $k \in \mathbb{N}$ and $\alpha \in(0,1)$. Let $\mathcal{C}^{k}\left(\mathbb{R}^{m}\right)$ (respectively $\mathcal{C}^{k, \alpha}\left(\mathbb{R}^{m}\right)$ ) be the set of $\mathcal{C}^{k}$ functions (respectively $\mathcal{C}^{k, \alpha}$ ) on $\mathbb{R}^{m}$. For $t \in \mathbb{R}^{+}$, define $\mathcal{C}^{t}\left(\mathbb{R}^{m}\right):=\mathcal{C}^{[t], t-[t]}\left(\mathbb{R}^{m}\right)$. Let $\mathcal{C}_{b}^{t}\left(\mathbb{R}^{m}\right)$ be the subset of $\mathcal{C}^{t}\left(\mathbb{R}^{m}\right)$ consisting of elements whose $\mathcal{C}^{t}$ norms are bounded.

Let $\Omega$ be a bounded open subset of $\mathbb{R}^{m}$ with smooth boundary. Let $\partial \Omega$ be its boundary. Then $\bar{\Omega}$ is a smooth compact manifold with boundary which is itself a global chart. We have the Banach spaces $\mathcal{C}^{t}(\bar{\Omega})$ and $\tilde{\mathcal{C}}^{t}(\bar{\Omega})$ as above. In what follows, we will give a description of the interpolation space

$$
\left(\tilde{\mathcal{C}}^{t_{0}}(\bar{\Omega}), \tilde{\mathcal{C}}^{t_{2}}(\bar{\Omega})\right)_{\alpha, \infty}
$$

for $0 \leq t_{0}<t_{2}<\infty$. The corresponding interpolation spaces for $\mathcal{C}^{t}(\bar{\Omega})$ and $\mathcal{C}_{b}^{t}\left(\mathbb{R}^{m}\right)$ are already known, see Theorems 2.7.2 and 4.5.2 in [23].

It should be noted that the spaces $\left(\mathcal{C}^{t_{0}}(\bar{\Omega}), \mathcal{C}^{t_{2}}(\bar{\Omega})\right)_{\alpha, \infty}$ are easily determined by using the result mentioned above for $\mathcal{C}_{b}^{t}\left(\mathbb{R}^{m}\right)$ and the fact that the restriction from $\mathcal{C}_{b}^{t}\left(\mathbb{R}^{m}\right)$ to $\mathcal{C}^{t}(\bar{\Omega})$ is a retraction, see [23, Th. 4.5.1]. Nevertheless, this property is no longer true if we replace $\mathcal{C}^{t}(\bar{\Omega})$ by $\tilde{\mathcal{C}}^{t}(\bar{\Omega})$ because even the restriction map from $\mathcal{C}_{b}^{t}\left(\mathbb{R}^{m}\right)$ to $\tilde{\mathcal{C}}^{t}(\bar{\Omega})$ is not well-defined. In order to compute (2.7), we will follow the original strategy for $\mathcal{C}_{b}^{t}\left(\mathbb{R}^{m}\right)$ in [23], see also [17]. Although, in essence, our below results can be implicitly deduced from [23], we will present them in a simplified and detailed way which is therefore accessible for a wider audience.

The following lemma is well-known but for the reader's convenience, a complete proof will be given.

Lemma 2.4. For every $t \in[0, \infty)$, every $f \in \mathcal{C}^{t}(\bar{\Omega})$ can be extended to be a function $E f \in$ $\mathcal{C}^{t}\left(\mathbb{R}^{m}\right)$ such that $\|E f\|_{\mathcal{C}^{t}\left(\mathbb{R}^{m}\right)} \leq C\|f\|_{\mathcal{C}^{t}(\bar{\Omega})}$, where $C$ is a constant independent of $f$.

Proof. We will use a reflexion argument. By using a partition of unity subordinated to a suitable finite covering of $\bar{\Omega}$, we can suppose that $\bar{\Omega}=\mathbb{R}^{m-1} \times \mathbb{R}^{+}$. Let $f \in \mathcal{C}^{t}\left(\mathbb{R}^{m-1} \times \mathbb{R}^{+}\right)$. Let $[t]$ be the integer part of $t$. Let $a_{1}, \cdots, a_{[t]+1}$ be real numbers which are chosen later. Define $E f:=f$ on $\mathbb{R}^{m-1} \times \mathbb{R}^{+}$and

$$
E f\left(x_{1}, \cdots, x_{n}\right):=\sum_{k=1}^{[t]+1} a_{k} f\left(x_{1}, \cdots, x_{n-1},-k x_{n}\right)
$$


otherwise. It is easy to see that $E f$ is continuous on $\mathbb{R}^{m}$. Now we will choose $a_{k}$ such that $E f \in \mathcal{C}^{[t]}$. If we can do so, we also get $E f \in \mathcal{C}^{t}$ because $D^{[t]} E f$ is $\mathcal{C}^{t-[t]}$ on $\mathbb{R}^{m-1} \times \mathbb{R}^{+}$, hence, on the whole $\mathbb{R}^{m}$ by its defining formula. One only needs to be concerned with the $x_{n}$-direction. Direct computations show that

$$
\partial_{x_{n}}^{l} E f\left(x_{1}, \cdots, x_{n-1}, 0\right)=\left(\sum_{k=1}^{[t]+1}(-k)^{l} a_{k}\right) \partial_{x_{n}}^{l} f\left(x_{1}, \cdots, x_{n-1}, 0\right),
$$

for $0 \leq l \leq[t]$. The regularity condition on $E f$ is equivalent to the linear system $\sum_{k=1}^{[t]+1}(-k)^{l} a_{k}=1$ for $0 \leq l \leq[t]$. Its determinant is a Vandermonde one. Hence the system has a unique solution $\left(a_{1}, \cdots, a_{[t]+1}\right)$. When $\left.f\right|_{\partial \Omega}=0$, it is clear from the defining formula of $E f$ that $\left.E f\right|_{\partial \Omega}=0$. The proof is finished.

Proposition 2.5. For every $\alpha \in(0,1)$ and every $k \in \mathbb{N}^{*}$, we have

$$
\left(\tilde{\mathcal{C}}^{0}(\bar{\Omega}), \tilde{\mathcal{C}}^{k}(\bar{\Omega})\right)_{\alpha, \infty} \supset \tilde{\mathcal{C}}^{\alpha k}(\bar{\Omega}),
$$

where the last inclusion means a continuous inclusion between Banach spaces.

Proof. Let $f \in \tilde{\mathcal{C}}^{\alpha k}(\bar{\Omega})$. Put $t:=\alpha k$. We write below $\lesssim$ to indicate $\leq$ up to a constant independent of $(f, \epsilon)$. By Lemma 2.4, we can extend $f$ to be a function $F$ in $\mathcal{C}^{t}\left(\mathbb{R}^{m}\right)$ with

$$
\|F\|_{\mathcal{C}^{t}\left(\mathbb{R}^{m}\right)} \leq C\|f\|_{\tilde{\mathcal{C}}^{t}(\bar{\Omega})},
$$

for some constant $C$ independent of $f$ and $\left.F\right|_{\partial \Omega}=0$. Let $\mathbb{B}_{r}$ denotes the ball of radius $r>0$ centered at 0 in $\mathbb{R}^{m}$ and $\mathbb{B}_{r}^{+}$denotes the subset of $\mathbb{B}_{r}$ consisting of $\mathbf{x}=\left(x_{1}, \cdots, x_{n}\right)$ with $x_{n} \geq 0$. Since $\bar{\Omega}$ is compact, we can cover $\partial \Omega$ by a finite number of small open subsets $\left\{U_{j}\right\}_{1 \leq j \leq N}$ of $\mathbb{R}^{m}$ such that in each $U_{j}$, by a suitable change of coordinates $\Psi_{j}$, we have

$$
\Psi_{j}\left(\bar{\Omega} \cap U_{j}\right)=\mathbb{B}_{2}^{+}
$$

and $\Psi_{j}\left(\partial \Omega \cap U_{j}\right)=\mathbb{B}_{2}^{+} \cap\left\{x_{n} \geq 0\right\}$. Without loss of generality, we can suppose that $\Psi_{j}^{-1}\left(\mathbb{B}_{1}^{+}\right)$also covers $\partial \Omega$. Put

$$
U_{0}:=\Omega \backslash \cup_{1 \leq j \leq N} \Psi_{j}^{-1}\left(\overline{\mathbb{B}}_{1}^{+}\right) .
$$

The family $\left\{U_{j}\right\}_{0 \leq j \leq N}$ covers $\bar{\Omega}$. Let $\left\{\chi_{j}\right\}_{0 \leq j \leq N}$ be smooth functions of $\mathbb{R}^{m}$ such that $0 \leq \chi_{j} \leq 1$ for $0 \leq j \leq N$, and $\operatorname{supp} \chi_{j} \Subset \Psi_{j}^{-1}\left(\mathbb{B}_{5 / 4}\right)$ for $1 \leq j \leq N$ and supp $\chi_{0} \Subset U_{0}$, and $\sum_{0 \leq j \leq N} \chi_{j}=1$ on $\bar{\Omega}$.

Define $F_{j}:=\left(\chi_{j} F\right) \circ \Psi_{j}^{-1}$. By the properties of $\left(\Psi_{j}, F\right)$ mentioned above, we have $\left.F_{j}\right|_{x_{n}=0}=0$. Let $\chi$ be a nonnegative smooth function on $\mathbb{R}^{m}$ which is compactly supported on $\mathbb{B}_{1}$ such that $\int_{\mathbb{R}^{m}} \chi d \mathbf{x}=1$. Taylor's expansion for $F_{j}$ gives

$$
F_{j}(\mathbf{x})=F_{j}(\mathbf{x}-\mathbf{y})+D F_{j}(\mathbf{x}-\mathbf{y}) \mathbf{y}+\cdots+\frac{1}{[t] !} D^{[t]} F_{j}(\mathbf{x}-\mathbf{y}) \mathbf{y}^{[t]}+R_{j}(\mathbf{x}, \mathbf{y}) \mathbf{y}^{[t]},
$$

where $R_{j}(\mathbf{x}, \mathbf{y})$ is, for $\mathbf{x}$ fixed, a $\mathcal{C}^{t-[t]}$ linear functional on $\left(\mathbb{R}^{m}\right)^{[t]}$ and we have

$$
R_{j}(\mathbf{x}, 0)=0, \quad\left\|R_{j}\right\|_{\mathcal{C}^{t-[t]}} \lesssim\left\|F_{j}\right\|_{\mathcal{C}^{t}} \leq C\|f\|_{\mathcal{C}^{t}} .
$$

Hence, one gets

$$
\left|R_{j}(\mathbf{x}, \mathbf{y})\right| \lesssim|\mathbf{y}|^{t-[t]}\|f\|_{\mathcal{C}^{t}} .
$$


Put

$$
\epsilon_{0}:=\min \left\{1 / 4, \operatorname{dist}\left(U_{0}, \partial \Omega\right)\right\} .
$$

Let $\epsilon \in\left(0, \epsilon_{0}\right)$. For $0 \leq j \leq N$, we define

$$
F_{j, \epsilon}(\mathbf{x}):=\int_{\mathbb{R}^{m}}\left[F_{j}(\mathbf{x}-\epsilon \mathbf{y})+D F_{j}(\mathbf{x}-\epsilon \mathbf{y})(\epsilon \mathbf{y})+\cdots+\frac{1}{[t] !} D^{[t]} F_{j}(\mathbf{x}-\epsilon \mathbf{y})(\epsilon \mathbf{y})^{[t]}\right] \chi(\mathbf{y}) d \mathbf{y} .
$$

Observe that $F_{0, \epsilon}$ is a smooth function in $\tilde{\mathcal{C}}^{\infty}(\bar{\Omega})$ by the choice of $\epsilon$ and $F_{j, \epsilon}$ is smooth on $\mathbb{R}^{m}$ and compactly supported on $\mathbb{B}_{3 / 2}$ for $1 \leq j \leq N$. A property of the convolution implies that $F_{j, \epsilon}$ converges to $F_{j}$ in $\mathcal{C}^{0}$-topology. Precisely, using (2.9), (2.11) and (2.10) yields that

$$
\left|F_{j, \epsilon}(\mathbf{x})-F_{j}(\mathbf{x})\right| \leq \epsilon^{[t]} \int_{\mathbb{R}^{m}}\left|R_{j}(\mathbf{x}, \epsilon \mathbf{y})\right| \chi(\mathbf{y}) d \mathbf{y} \leq C \epsilon^{t}\|f\|_{\mathcal{C}^{t}},
$$

for every $\mathbf{x}$. Let $\tau$ be a smooth function on $\mathbb{R}$ compactly supported on $[-2,2]$ such that $\tau \equiv 1$ on $[-3 / 2,3 / 2]$. Define

$$
F_{j, \epsilon}^{\prime}\left(x_{1}, \cdots, x_{n}\right):=F_{j, \epsilon}\left(x_{1}, \cdots, x_{n-1}, x_{n}\right)-\tau\left(x_{n}\right) F_{j, \epsilon}\left(x_{1}, \cdots, x_{n-1}, 0\right),
$$

for $1 \leq j \leq N$ and we put $F_{0, \epsilon}^{\prime}:=F_{0, \epsilon}$ for consistence. We immediately see that $F_{j, \epsilon}^{\prime}=0$ on $\left\{x_{n}=0\right\}$ and $\operatorname{supp} F_{j, \epsilon}^{\prime} \subset \mathbb{B}_{2}$. As a consequence, $F_{j, \epsilon}^{\prime} \circ \Psi_{j}$ is smooth on $\mathbb{R}^{m}$ and vanishes on $\partial \Omega$. We deduce from (2.12) and the fact that $\left.F_{j}\right|_{\left\{x_{n}=0\right\}} \equiv 0$ that

$$
\begin{aligned}
& \left|F_{j, \epsilon}^{\prime}(\mathbf{x})-F_{j}(\mathbf{x})\right| \leq\left|F_{j, \epsilon}(\mathbf{x})-F_{j}(\mathbf{x})\right|+ \\
& \quad\left|F_{j, \epsilon}\left(x_{1}, \cdots, x_{n-1}, 0\right)-F_{j}\left(x_{1}, \cdots, x_{n-1}, 0\right)\right| \leq 2 C \epsilon^{t}\|f\|_{\mathcal{C}^{t}} .
\end{aligned}
$$

Define

$$
g_{1, \epsilon}:=\left.\sum_{0 \leq j \leq N} F_{j, \epsilon}^{\prime} \circ \Psi_{j}\right|_{\bar{\Omega}} \in \tilde{\mathcal{C}}^{\infty}(\bar{\Omega})
$$

and $g_{0, \epsilon}:=f-g_{1, \epsilon} \in \tilde{\mathcal{C}}^{0}(\bar{\Omega})$. We have $f=g_{0, \epsilon}+g_{1, \epsilon}$. In view of (2.6), we have to estimate $\left\|g_{0, \epsilon}\right\|_{\tilde{\mathcal{C}}^{0}(\bar{\Omega})}$ and $\left\|g_{1, \epsilon}\right\|_{\tilde{\mathcal{C}}^{k}(\bar{\Omega})}$. Since $f=\sum_{0 \leq j \leq N} F_{j} \circ \Psi_{j}$, we have

$$
g_{0, \epsilon}=\sum_{0 \leq j \leq N}\left(F_{j} \circ \Psi_{j}-F_{j, \epsilon}^{\prime} \circ \Psi_{j}\right) .
$$

Taking into account (2.13), one gets

$$
\left\|g_{0, \epsilon}\right\|_{\tilde{\mathcal{C}}^{0}(\bar{\Omega})} \lesssim \epsilon^{t}\|f\|_{\tilde{\mathcal{C}}^{t}(\bar{\Omega})} .
$$

For $0 \leq l \leq[t]$, we define

$$
G_{j, l}(\mathbf{x}, \mathbf{y}):=D^{l} F_{j}(\mathbf{y})+D^{l+1} F_{j}(\mathbf{y}) \mathbf{y}+\cdots+\frac{1}{([t]-l) !} D^{[t]} F_{j}(\mathbf{y})(\mathbf{x}-\mathbf{y})^{[t]-l}
$$

which is the Taylor expansion up to the $([t]-l)$ order of $D^{l} F_{j}(\mathbf{x})$ at $\mathbf{y}$. Thus arguing as in (2.10), we get

$$
\left|G_{j, l}(\mathbf{x}, \mathbf{y})-D^{l} F_{j}(\mathbf{x})\right| \lesssim\|f\|_{\mathcal{C}^{t}}|\mathbf{y}|^{t-l} .
$$

The equality (2.11) can be rewritten as

$$
F_{j, \epsilon}(\mathbf{x})=\epsilon^{-m} \int_{\mathbb{R}^{m}}\left[F_{j}\left(\mathbf{y}^{\prime}\right)+D F_{j}\left(\mathbf{y}^{\prime}\right)\left(\mathbf{x}-\mathbf{y}^{\prime}\right)+\cdots+\frac{1}{[t] !} D^{[t]} F_{j}\left(\mathbf{y}^{\prime}\right)\left(\mathbf{x}-\mathbf{y}^{\prime}\right)^{[t]}\right] \chi\left(\frac{\mathbf{x}-\mathbf{y}^{\prime}}{\epsilon}\right) d \mathbf{y}^{\prime} .
$$


Differentiating the last equality in $\mathrm{x}$ for $k^{\prime}$ times gives

$$
\begin{aligned}
D_{\mathbf{x}}^{k^{\prime}} F_{j, \epsilon}(\mathbf{x}) & =\epsilon^{-m-k^{\prime}+l} \sum_{0 \leq l \leq \min \left\{k^{\prime},[t]\right\}} \int_{\mathbb{R}^{m}} G_{j, l}\left(\mathbf{x}, \mathbf{y}^{\prime}\right) \otimes D^{k^{\prime}-l} \chi\left(\frac{\mathbf{x}-\mathbf{y}^{\prime}}{\epsilon}\right) d \mathbf{y}^{\prime} \\
& =\epsilon^{-k^{\prime}+l} \sum_{0 \leq l \leq \min \left\{k^{\prime},[t]\right\}} \int_{\mathbb{R}^{m}} G_{j, l}(\mathbf{x}, \mathbf{x}-\epsilon \mathbf{y}) \otimes D^{k^{\prime}-l} \chi(\mathbf{y}) d \mathbf{y}
\end{aligned}
$$

by a suitable change of coordinates. Since $\int_{\mathbb{R}^{m}} D_{\mathbf{x}}^{l} \chi(\mathbf{y}) d \mathbf{y}=0$ for any $l \geq 1$, we obtain

$$
\int_{\mathbb{R}^{m}} G_{j, l}(\mathbf{x}, \mathbf{x}-\epsilon \mathbf{y}) \otimes D^{k^{\prime}-l} \chi(\mathbf{y}) d \mathbf{y}=\int_{\mathbb{R}^{m}}\left(G_{j, l}(\mathbf{x}, \mathbf{x}-\epsilon \mathbf{y})-D^{l} F_{j}(\mathbf{x})\right) \otimes D^{k^{\prime}-l} \chi(\mathbf{y}) d \mathbf{y}
$$

which is of absolute value $\lesssim \epsilon^{t-l}\|f\|_{\mathcal{C}^{t}}$ by using (2.15) and the fact that supp $\chi \subset \mathbb{B}_{1}$. Combining (2.16) with (2.17) gives

$$
\left|D_{\mathbf{x}}^{k^{\prime}} F_{j, \epsilon}(\mathbf{x})\right| \lesssim \epsilon^{-k^{\prime}+t}\|f\|_{\mathcal{C}^{t}}
$$

which implies that

$$
\left\|g_{1, \epsilon}\right\|_{\tilde{\mathcal{C}}^{k}(\bar{\Omega})} \lesssim \epsilon^{-k+t}\|f\|_{\mathcal{C}^{t}}
$$

by choosing $k^{\prime}=k$. Taking into account (2.14), (2.18) and (2.5), one deduces that

$$
\epsilon^{-\alpha k} K\left(\epsilon^{k}, f ; \tilde{\mathcal{C}}^{0}(\bar{\Omega}), \tilde{\mathcal{C}}^{k}(\bar{\Omega})\right) \leq \epsilon^{t}\left(\left\|g_{0, \epsilon}\right\|_{\tilde{\mathcal{C}}^{0}}+\epsilon^{k}\left\|g_{1, \epsilon}\right\|_{\tilde{\mathcal{C}}^{k}}\right) \lesssim\|f\|_{\tilde{\mathcal{C}}^{t}(\bar{\Omega})},
$$

for every $\epsilon \in\left(0, \epsilon_{0}\right)$. When $\epsilon \geq \epsilon_{0}$, since

$$
f=f+0 \in \tilde{\mathcal{C}}^{0}(\bar{\Omega})+\tilde{\mathcal{C}}^{1}(\bar{\Omega})
$$

we have

$$
\epsilon^{-\alpha k} K\left(\epsilon^{k}, f ; \tilde{\mathcal{C}}^{0}(\bar{\Omega}), \tilde{\mathcal{C}}^{k}(\bar{\Omega})\right) \leq \epsilon_{0}^{-\alpha k}\|f\|_{\mathcal{\mathcal { C }}^{0}(\bar{\Omega})} \leq \epsilon_{0}^{-\alpha k}\|f\|_{\mathcal{\mathcal { C }}^{\alpha k}(\bar{\Omega})} .
$$

Hence, $f \in\left(\tilde{\mathcal{C}}^{0}(\bar{\Omega}), \tilde{\mathcal{C}}^{k}(\bar{\Omega})\right)_{\alpha, \infty}$. The proof is finished.

For every $h \in \mathbb{R}^{m}$ and every a function $g$ on $\mathbb{R}^{m}$, define the operator

$$
\Delta_{h} g(x):=g(x+h)-g(x)
$$

for every $x \in \mathbb{R}^{m}$. The following property is crucial for the next proposition.

Lemma 2.6. Let $\alpha \in(0,1)$ and $l$ be an integer $\geq 1$. For $g \in \mathcal{C}_{b}^{\alpha}\left(\mathbb{R}^{m}\right)$, we put

$$
\|g\|_{\alpha, \Delta, l}:=\|g\|_{\mathcal{C}^{0}}+\sup _{x, h \in \mathbb{R}^{m}, h \neq 0} \frac{\left|\Delta_{h}^{l} g\right|}{|h|^{\alpha}} .
$$

Then the last formula defines a norm on $\mathcal{C}_{b}^{\alpha}\left(\mathbb{R}^{m}\right)$ which is equivalent to its usual $\mathcal{C}^{\alpha}$ norm. More precisely, there exists a positive constant $C_{l, \alpha}$ depending only on $(l, \alpha)$ such that for every $g$, we have

$$
C_{l, \alpha}^{-1}\|g\|_{\mathcal{C}^{\alpha}} \leq\|g\|_{\alpha, \Delta, l} \leq C_{l, \alpha}\|g\|_{\mathcal{C}^{\alpha}} .
$$

Proof. This is a simplification of Lemma 1.13.4 in [23]. When $l=1$, the two norms are identical. Consider $l \geq 2$. Observe that it is enough to prove the desired result for $l=2$ because the general case can easily follow by induction. It is clear that $\|g\|_{\alpha, \Delta, 2} \leq 2\|g\|_{\mathcal{C}^{\alpha}}$. We now prove the converse inequality. The key argument is the following formula:

$$
g(x+h)-g(x)=\frac{1}{2}(g(x+2 h)-g(x))-\frac{g(x+2 h)-2 g(x+h)+g(x)}{2} .
$$


Dividing the last equality by $|h|^{\alpha}$ gives

$$
\frac{|g(x+h)-g(x)|}{|h|^{\alpha}} \leq 2^{\alpha-1} \frac{|g(x+2 h)-g(x)|}{|2 h|^{\alpha}}+\frac{|g(x+2 h)-2 g(x+h)+g(x)|}{2|h|^{\alpha}} .
$$

By taking the supremum over $\left\{(x, h) \in \mathbb{R}^{2 m}, h \neq 0\right\}$ in the last inequality, we deduce that

$$
\|g\|_{\mathcal{C}^{\alpha}} \leq 2^{\alpha-1}\|g\|_{\mathcal{C}^{\alpha}}+\|g\|_{\alpha, \Delta, 2} \text {. }
$$

Since $2^{\alpha-1}<1$ we get the desired conclusion. The proof is finished.

Proposition 2.7. Let $k$ be a positive integer and let $\alpha$ be a real number in $(0,1)$. Assume that $\alpha k \in(0,1)$. Then we have

$$
\left(\tilde{\mathcal{C}}^{0}(\bar{\Omega}), \tilde{\mathcal{C}}^{k}(\bar{\Omega})\right)_{\alpha, \infty} \subset \tilde{\mathcal{C}}^{\alpha k}(\bar{\Omega})
$$

Proof. Let take an element $f \in\left(\tilde{\mathcal{C}}^{0}(\bar{\Omega}), \tilde{\mathcal{C}}^{k}(\bar{\Omega})\right)_{\alpha, \infty}$. Suppose that $f=g_{0}+g_{1}$ with $g_{0} \in$ $\tilde{\mathcal{C}}^{0}(\bar{\Omega})$ and $g_{1} \in \tilde{\mathcal{C}}^{k}(\bar{\Omega})$. We have $\Delta_{h}^{k} f=\Delta_{h}^{k} g_{0}+\Delta_{h}^{k} g_{1}$. By using Taylor's expansion of $g_{1}$, observe that $\left|\Delta_{h}^{k} g_{1}\right| \leq C|h|^{k}\left\|g_{1}\right\|_{\mathcal{C}^{k}}$ for some constant $C$ independent of $\left(g_{1}, h\right)$. On the other hand, $\left|\Delta_{h}^{k} g_{0}\right| \leq 2^{l}\left\|g_{0}\right\|_{\mathcal{C}^{0}}$. Combining these inequalities gives

$$
\left|\Delta_{h}^{k} f\right| \leq 2^{l}\|g\|_{\mathcal{C}^{0}}+C|h|^{k}\left\|g_{1}\right\|_{\mathcal{C}^{k}} \lesssim\|g\|_{\mathcal{C}^{0}}+|h|^{k}\left\|g_{1}\right\|_{\mathcal{C}^{k}}
$$

for every $\left(g_{0}, g_{1}\right)$ with $f=g_{0}+g_{1}$. Taking the infimum in the last inequality in $\left(g_{0}, g_{1}\right)$, we obtain

$$
\left|\Delta_{h}^{k} f\right| \lesssim K\left(h^{k}, f ; \tilde{\mathcal{C}}^{0}(\bar{\Omega}), \tilde{\mathcal{C}}^{1}(\bar{\Omega})\right) \leq|h|^{\alpha k}\|f\|_{\left(\tilde{\mathcal{C}}^{0}(\bar{\Omega}), \tilde{\mathcal{C}}^{k}(\bar{\Omega})\right)_{\alpha, \infty}}
$$

As a consequence, one gets

$$
\|f\|_{\alpha k, \Delta, k} \lesssim\|f\|_{\left(\tilde{\mathcal{C}}^{0}(\bar{\Omega}), \tilde{\mathcal{C}}^{k}(\bar{\Omega})\right)_{\alpha, \infty}} .
$$

By Lemma 2.6 and the hypothesis that $\alpha k<1$, we obtain the desired result. The proof is finished.

Corollary 2.8. For every $\alpha \in(0,1)$, every real nonnegative numbers $t_{1}$ and $t_{2}$, we have

$$
\left(\tilde{\mathcal{C}}^{t_{1}}(\bar{\Omega}), \tilde{\mathcal{C}}^{t_{2}}(\bar{\Omega})\right)_{\alpha, \infty} \supset \tilde{\mathcal{C}}^{\alpha t_{2}+(1-\alpha) t_{1}}(\bar{\Omega}) .
$$

Proof. For simplicity, we give a proof for $t_{2}=k \in \mathbb{N}^{*}$ and $t_{1}=\beta \in[0,1)$. The general case can be deduced by using similar arguments. By a consequence of the reiteration theorem (see [17, Re. 1.3.7]), we have the following general formula:

$$
\left(\left(A_{0}, A_{1}\right)_{\theta, \infty}, A_{1}\right)_{\alpha, \infty}=\left(A_{0}, A_{1}\right)_{(1-\alpha) \theta+\alpha, \infty} .
$$

Applying the last equality to

$$
A_{0}=\tilde{\mathcal{C}}^{0}(\bar{\Omega}), A_{1}=\tilde{\mathcal{C}}^{k}(\bar{\Omega}) \quad \text { and } \quad \theta=\beta / k
$$

and using the fact that $\left(A_{0}, A_{1}\right)_{\theta, \infty}=\tilde{\mathcal{C}}^{\beta}(\bar{\Omega})$ (by Proposition 2.7 and 2.5), we obtain the desired inclusion. The proof is finished.

Since $(\mathbb{R}, \mathbb{R})_{\alpha, \infty}=\mathbb{R}$ for any $\alpha \in(0,1)$, applying Theorem 2.3 to

$$
A_{0}=\tilde{\mathcal{C}}^{t_{0}}(\bar{\Omega}), \quad A_{1}=\tilde{\mathcal{C}}^{t_{2}}(\bar{\Omega}), \quad B_{0}=B_{1}=\mathbb{R},
$$

and then using Corollary 2.8 , we obtain the following result. 
Corollary 2.9. Let $\Omega$ be a bounded open subset of $\mathbb{R}^{m}$ with smooth boundary. Let $t_{0}, t_{1}$ and $t_{2}$ be three real numbers such that $0 \leq t_{0}<t_{1}<t_{2}$. Let $S$ be a bounded linear map from $\tilde{\mathcal{C}}^{t_{0}}(\bar{\Omega})$ to $\mathbb{R}$. Then the restriction $\left.S\right|_{\tilde{\mathcal{C}}^{t_{j}}(\bar{\Omega})}$ of $S$ to $\tilde{\mathcal{C}}^{t_{j}}(\bar{\Omega})$ for $j=1$ or 2 is also a bounded linear map from $\tilde{\mathcal{C}}^{t_{j}}(\bar{\Omega})$ to $\mathbb{R}$ and

$$
\left\|\left.S\right|_{\tilde{\mathcal{C}}^{t_{1}(\bar{\Omega})}}\right\| \leq\left. c\left\|\left.S\right|_{\tilde{\mathcal{C}}^{t_{0}(\bar{\Omega})}}||^{t_{*}}\right\| S\right|_{\tilde{\mathcal{C}}^{t_{2}(\bar{\Omega})}} \|^{1-t_{*}},
$$

where $c$ is a constant independent of $S$ and $t_{*}$ is the unique real number for which $t_{1}=$ $t_{*} t_{0}+\left(1-t_{*}\right) t_{2}$.

\section{ANALYTIC DISCS PARTLY ATTACHED TO A GENERIC SUBMANIFOLD}

Firstly we fix some notations which will be valid throughout the rest of paper. For every Riemannian smooth manifold $Y$, any $a \in Y$ and $r \in \mathbb{R}^{+}$, we denote by $\mathbb{B}_{Y}(a, r)$ the ball of radius $r$ centered at $a$ of $Y$ and by ool $_{Y}$ the Riemannian volume form of $Y$. When $Y=\mathbb{R}^{m}$ for some $m \in \mathbb{N}$ with the Euclidean metric, we write $\mathbb{B}_{m}(a, r)$ instead of $\mathbb{B}_{Y}(a, r)$ and $\mathbb{B}_{m}$ instead of $\mathbb{B}_{m}(0,1)$. In particular, when $Y=\mathbb{C} \simeq \mathbb{R}^{2}$ and $a=0$, we put $\mathbb{D}_{r}:=\mathbb{B}_{2}(0, r)$ and $\mathbb{D}:=\mathbb{B}_{2}(0,1)$. For every $m \in \mathbb{N}^{*}$, we identify $\mathbb{C}^{m}$ with $\mathbb{R}^{2 m}$ via the formula $\mathbb{C}^{m}=\mathbb{R}^{m}+i \mathbb{R}^{m}$.

Let $\partial \mathbb{D}$ be the boundary of $\mathbb{D}$ and $\partial^{+} \mathbb{D}:=\{\xi \in \mathbb{D}: \operatorname{Re} \xi \geq 0\}$. We sometimes identify $\xi \in \mathbb{D}$ with $\theta \in(-\pi, \pi]$ by letting $\xi=e^{i \theta}$. An analytic disc $f$ in $X$ is a holomorphic mapping from $\mathbb{D}$ to $X$ which is continuous up to the boundary $\partial \mathbb{D}$ of $\mathbb{D}$. For an interval $I \subset \partial \mathbb{D}, f$ is said to be $I$-attached to a subset $E \subset X$ if $f(I) \subset E$. When $I=\partial^{+} \mathbb{D}$, an analytic disc $I$-attached to $E$ is said to be half-attached to $E$.

Let $K$ be a generic immersed $\mathcal{C}^{3}$ submanifold of $X$. Observe that the dimension of $K$ is at least $n$. Throughout the paper, we only consider the case where $\operatorname{dim} K=n$, hence its codimension $d$ equals $n$. This is in fact the most interesting case and the general case will be easily deduced from it. In Section 5 , we will explain the necessary modifications to get Theorem 1.4 when $\operatorname{dim} K>n$.

Our goal is to for each $a \in K$ construct a $\mathcal{C}^{2,1 / 2}$-differentiable family of analytic discs partly attached to $K$ which covers an open neighborhood of $a$ in $X$. It should be noted that any family of discs partly attached to $K$ degenerates near $K$ due to its attachment to $K$. Controlling such behaviour around $K$ is actually the key point in this section. We also need that the part of this family lying in $K$ must cover an open neighborhood of 0 in $K$. Constructing analytic discs is an important tool in Cauchy-Riemann geometry. Generally, one uses a suitable Bishop-type equation together with a choice of initial data depending on situations to obtain the desired result. The reader may also consult [1, 18, 19] and references therein for more information. In what follows, we will apply the same strategy combining with the ideas from [24].

The following local coordinates are frequently used in the Cauchy-Riemann geometry.

Lemma 3.1. Through every point a of $K$, there exist local holomorphic coordinates $(W, \mathbf{z})$ of $X$ around a such that in that local coordinates, the point $a$ is the origin and $K \cap W$ is the graph over $\mathbb{B}_{n}$ of a $\mathcal{C}^{3}$ map $h$ from $\overline{\mathbb{B}}_{n}$ to $\mathbb{R}^{n}$ which satisfies $D^{j} h(0)=0$ with $j=0,1,2$, where Dh denotes the differential of $h$. Moreover, $\|h\|_{\mathcal{C}^{3}}$ is bounded uniformly in $a \in \tilde{K}$.

Proof. The existence of such $h$ with $h(0)=D h(0)=0$ is well-known, see [1] for example. In order to obtain the additional property $D^{2} h(0)=0$, one will need to perform a change of coordinates, we refer to [18, Sec. 6.10] for details. The proof is finished. 
From now on, fix an arbitrary point $a \in K$ and we confine ourselves to the local chart described in Lemma 3.1. In other words, we will work on $\mathbb{C}^{n}$ and

$$
K^{\prime}:=\left\{\mathbf{z}=\mathbf{x}+i h(\mathbf{x}) \in \mathbb{C}^{n}: \mathbf{x} \in \mathbb{B}_{n}\right\},
$$

where we have $h(0)=D h(0)=0$. For most of the time, the last condition is enough for our purposes, we will only need $D^{2} h(0)=0$ in the proof of Proposition 4.5. The property of $h$ yields that there is a constant $c_{0}$ for which

$$
|h(\mathbf{x})| \leq c_{0}|\mathbf{x}|^{2}, \quad|D h(\mathbf{x})| \leq c_{0}|\mathbf{x}|,
$$

for every $\mathbf{x} \in \overline{\mathbb{B}}_{n}$.

In this paragraph, we prepare some useful facts about harmonic functions on the unit disc which will be indispensable for studying Bishop-type equations later. Denote by $z=x+i y$ the complex variable on $\mathbb{C}$ and by $\xi=e^{i \theta}$ the variable on $\partial \mathbb{D}$. Let $u_{0}(\xi)$ be an arbitrary continuous function on $\partial \mathbb{D}$. Recall that $u_{0}$ can be extended uniquely to be a harmonic function on $\mathbb{D}$ which is continuous on $\overline{\mathbb{D}}$. Since this correspondence is bijective, without stating explicitly, we will freely identify $u_{0}$ with its harmonic extension on $\mathbb{D}$. We will write $u_{0}(z)=u_{0}(x+i y)$ to indicate the harmonic extension of $u_{0}\left(e^{i \theta}\right)$. It is well-known that the Cauchy transform of $u_{0}$, given by

$$
\mathcal{C} u_{0}(z):=\frac{1}{2 \pi} \int_{-\pi}^{\pi} u_{0}\left(e^{i \theta}\right) \frac{e^{i \theta}+z}{e^{i \theta}-z} d \theta
$$

is a holomorphic function on $\mathbb{D}$ whose real part is $u_{0}$. Let $\mathcal{T} u_{0}$ be the imaginary part of $\mathcal{C} u_{0}$. Decomposing the last formula into the real and imaginary parts, we obtain

$$
u_{0}(z)=\frac{1}{2 \pi} \int_{-\pi}^{\pi} \frac{\left(1-|z|^{2}\right)}{\left|e^{i \theta}-z\right|^{2}} u_{0}\left(e^{i \theta}\right) d \theta .
$$

and

$$
\mathcal{T} u_{0}(z)=\frac{1}{2 \pi} \int_{-\pi}^{\pi} \frac{\left(z e^{-i \theta}-\bar{z} e^{i \theta}\right)}{i\left|e^{i \theta}-z\right|^{2}} u_{0}\left(e^{i \theta}\right) d \theta .
$$

The function $\mathcal{T} u_{0}$ is harmonic on $\mathbb{D}$ but is not always continuous up to the boundary of $\mathbb{D}$. Let $k$ be an arbitrary natural number and let $\beta$ be an arbitrary number in $(0,1)$. A result of Privalov (see [18, Th. 4.12]) implies that if $u_{0}$ belongs to $\mathcal{C}^{k, \beta}(\partial \mathbb{D})$, then $\mathcal{T} u_{0}$ is continuous up to $\partial \mathbb{D}$ and $\left\|\mathcal{T} u_{0}\right\|_{\mathcal{C}^{k, \beta}(\partial \mathbb{D})}$ is bounded by $\left\|u_{0}\right\|_{\mathcal{C}^{k, \beta}(\partial \mathbb{D})}$ times a constant independent of $u_{0}$. Hence, the linear self-operator of $\mathcal{C}^{k, \beta}(\partial \mathbb{D})$ defined by sending $u_{0}$ to the restriction of $\mathcal{T} u_{0}$ onto $\partial \mathbb{D}$ is bounded and called the Hilbert transform. For simplicity, we also denote it by $\mathcal{T}$. For our later purposes, it is convenient to use a modified version $\mathcal{T}_{1}$ of $\mathcal{T}$ defined by

$$
\mathcal{T}_{1} u_{0}:=\mathcal{T} u_{0}-\mathcal{T} u_{0}(1)
$$

Hence we always have $\mathcal{T}_{1} u_{0}(1)=0$ and

$$
\partial_{\theta} \mathcal{T}_{1} u_{0}=\partial_{\theta} \mathcal{T} u_{0}=\mathcal{T} \partial_{\theta} u_{0}
$$

provided that $u_{0} \in \mathcal{C}^{1, \beta}(\partial \mathbb{D})$ with $\beta \in(0,1)$, see [18, p.121] for a proof. The boundedness of $\mathcal{T}$ on $\mathcal{C}^{k, \beta}(\partial \mathbb{D})$ implies that there is a constant $C_{k, \beta}>1$ such that for any $v \in \mathcal{C}^{k, \beta}(\partial \mathbb{D})$ we have

$$
\left\|\mathcal{T}_{1} v\right\|_{\mathcal{C}^{k, \beta}(\partial \mathbb{D})} \leq C_{k, \beta}\|v\|_{\mathcal{C}^{k, \beta}(\partial \mathbb{D})} .
$$


Extending $u_{0}, \mathcal{T}_{1} u_{0}$ harmonically to $\mathbb{D}$. By construction, the function $f(z):=-\mathcal{T}_{1} u_{0}(z)+$ $i u_{0}(z)$ is holomorphic on $\mathbb{D}$ and continuous on $\overline{\mathbb{D}}$ provided that $u_{0}$ is in $\mathcal{C}^{\beta}(\partial \mathbb{D})$ with $0<$ $\beta<1$. By [19, Th. 4.2], $\|f\|_{\mathcal{C}^{k, \beta}(\overline{\mathbb{D}})}$ is bounded by $\|f\|_{\mathcal{C}^{k, \beta}(\partial \mathbb{D})}$ times a constant depending only on $(k, \beta)$. Since $\left\|u_{0}\right\|_{\mathcal{C}^{k, \beta}(\overline{\mathbb{D}})} \leq\|f\|_{\mathcal{C}^{k, \beta}(\overline{\mathbb{D}})}$ and $\|f\|_{\mathcal{C}^{k, \beta}(\partial \mathbb{D})} \leq\left(1+C_{k, \beta}\right)\left\|u_{0}\right\|_{\mathcal{C}^{k, \beta}(\partial \mathbb{D})}$ by (3.4), we have

$$
\left\|u_{0}\right\|_{\mathcal{C}^{k, \beta}(\overline{\mathbb{D}})} \leq C_{k, \beta}^{\prime}\left\|u_{0}\right\|_{\mathcal{C}^{k, \beta}(\partial \mathbb{D})},
$$

for some constant $C_{k, \beta}^{\prime}$ depending only on $(k, \beta)$. A direct consequence of the above inequalities is that when $u_{0}$ is smooth on $\partial \mathbb{D}$, the associated holomorphic function $f$ is also smooth on $\overline{\mathbb{D}}$.

Lemma 3.2. There exist a function $u_{0} \in \mathcal{C}^{\infty}(\partial \mathbb{D})$ and two positive constants $\left(\theta_{u_{0}}, c_{u_{0}}\right)$ such that $u_{0}\left(e^{i \theta}\right)=0$ for $\theta \in\left[-\theta_{u_{0}}, \theta_{u_{0}}\right] \subset[-\pi / 2, \pi / 2]$ and $\partial_{x} u_{0}(1)=-1$ and $u_{0}(z)>c_{u_{0}}(1-|z|)$ for every $z \in \mathbb{D}$.

Proof. Let $u$ be a smooth function on $\partial \mathbb{D}$ vanishing on $\partial^{+} \mathbb{D}$. By Poisson's formula, we have

$$
u(z)=\frac{1}{2 \pi} \int_{-\pi}^{\pi} \frac{\left(1-|z|^{2}\right)}{\left|e^{i \theta}-z\right|^{2}} u\left(e^{i \theta}\right) d \theta .
$$

Differentiating (3.6) gives

$$
\partial_{x} u(1)=\frac{1}{2 \pi} \int_{-\pi}^{\pi} \frac{u\left(e^{i \theta}\right)}{\cos \theta-1} d \theta .
$$

Note that the last integral is well-defined because $u$ vanishes on $\partial^{+} \mathbb{D}$. It is easy to choose a smooth $u$ so that the above integral is equal to -1 and $u \equiv 0$ on $\partial^{+} \mathbb{D}$ and $u\left(e^{i \theta}\right)>0$ for $|\theta| \geq 3 \pi / 2$. The last property and (3.6) show that $u(z)>0$ for every $z \in \mathbb{D}$.

We have chosen $u$ with the property that $\partial_{x} u(1)=-1$ and $u(z)>0$ for $z \in \mathbb{D}$. This implies that $\partial_{x} u\left(e^{i \theta}\right) \leq-1 / 2$ for every $\theta \in\left[-\theta_{0}, \theta_{0}\right] \subset(-\pi / 2, \pi / 2)$ for $\theta_{0}$ close enough to 1. Since $u$ vanishes on $\partial^{+} \mathbb{D}$, we have

$$
0=\partial_{\theta} u\left(e^{i \theta}\right)=-\partial_{x} u\left(e^{i \theta}\right) \sin \theta+\partial_{y} u\left(e^{i \theta}\right) \cos \theta
$$

which yields

$$
\partial_{y} u\left(e^{i \theta}\right)=\partial_{x} u\left(e^{i \theta}\right) \tan \theta
$$

for $\theta \in\left[-\theta_{0}, \theta_{0}\right]$. Let $z=|z| e^{i \theta} \in \mathbb{D}$ such that $\theta \in\left[-\theta_{0}, \theta_{0}\right]$. Taylor's expansion for $u$ at $e^{i \theta}$ gives

$$
\begin{aligned}
u\left(|z| e^{i \theta}\right) & =u\left(e^{i \theta}\right)+(|z| \cos \theta-\cos \theta) \partial_{x} u\left(e^{i \theta}\right)+(|z| \sin \theta-\sin \theta) \partial_{y} u\left(e^{i \theta}\right)+O\left((1-|z|)^{2}\right) \\
& \left.=\frac{(|z|-1) \partial_{x} u\left(e^{i \theta}\right)}{\cos \theta}+O\left((1-|z|)^{2}\right) \quad \text { (by (3.7) }\right) .
\end{aligned}
$$

By our choice of $\theta_{0}$, the last equality gives

$$
u\left(|z| e^{i \theta}\right) \geq(1-|z|) / 2-\|u\|_{\mathcal{C}^{2}(\mathbb{D})}(1-|z|)^{2} \geq(1-|z|) / 4,
$$

for $|z| \geq 1-1 / 4\|u\|_{\mathcal{C}^{2}(\mathbb{D})}^{-1}$. When $|z| \leq 1-1 / 4\|u\|_{\mathcal{C}^{2}(\mathbb{D})}^{-1}$, we have $u(z) \geq c$ for some constant $c$ independent of $z$. This combined with the fact that $(1-|z|) \leq 1$ implies that there is a 
positive constant $c^{\prime}$ for which $u(z) \geq c^{\prime}(1-|z|)$ for $|z| \leq 1-1 / 4\|u\|_{\mathcal{C}^{2}(\mathbb{D})}^{-1}$. In summary, we can find a positive constant $c^{\prime}$ for which

$$
u(z) \geq c^{\prime}(1-|z|),
$$

for $z=|z| e^{i \theta} \in \mathbb{D}$ with $\theta \in\left[-\theta_{0}, \theta_{0}\right]$.

Now let $\Omega$ be a simply connected subdomain of $\mathbb{D}$ with smooth boundary such that $\Omega$ is strictly convex and $\bar{\Omega} \cap \overline{\mathbb{D}}=\left[e^{-i \theta_{0} / 2}, e^{i \theta_{0} / 2}\right]$. By Painvelé's theorem (see, for example, [2, Th. 3.1] or [16, Th. 5.3.8]), there is a smooth diffeomorphism $\Phi$ from $\overline{\mathbb{D}}$ to $\bar{\Omega}$ which is a biholomorphism from $\mathbb{D}$ to $\Omega$ and $\Phi(1)=1$. Define $u_{0}^{\prime}:=u \circ \Phi$ which is a smooth function on $\overline{\mathbb{D}}$ and harmonic on $\mathbb{D}$. We immediately have $u_{0}^{\prime}(z)>0$ on $\mathbb{D}$.

Since $\Phi(1)=1$ and $\Phi$ sends $\partial \mathbb{D}$ to $\partial \Omega$, there is a small positive constant $\theta^{\prime}$ such that $\Phi\left(\left[e^{-i \theta_{0}^{\prime}}, e^{i \theta_{0}^{\prime}}\right]\right)$ is contained in $\left[e^{-i \theta_{0} / 2}, e^{i \theta_{0} / 2}\right]$. This yields $u_{0}^{\prime}\left(e^{i \theta}\right)=0$ for $|\theta| \leq \theta_{0}^{\prime}$ and $\operatorname{Re}^{2} \Phi\left(e^{i \theta}\right)+\operatorname{Im}^{2} \Phi\left(e^{i \theta}\right)=1$ on $\left[e^{-i \theta_{0}^{\prime}}, e^{i \theta_{0}^{\prime}}\right]$. Differentiating the last inequality at $\theta^{\prime}=0$ gives

$$
\operatorname{Re} \Phi(1) \partial_{y} \operatorname{Re} \Phi(1)+\operatorname{Im} \Phi(1) \partial_{y} \operatorname{Im} \Phi(1)=0
$$

which combined with $\Phi(1)=1$ implies that $\partial_{y} \operatorname{Re} \Phi(1)=0$. The last equality coupled with the fact that $\Phi$ is holomorphic implies

$$
\operatorname{det} D_{(x, y)} \Phi(1)=\left(\partial_{x} \operatorname{Re} \Phi(1)\right)^{2}+\left(\partial_{y} \operatorname{Re} \Phi(1)\right)^{2}=\left(\partial_{x} \operatorname{Re} \Phi(1)\right)^{2} .
$$

As a result, we have $\partial_{x} \operatorname{Re} \Phi(1) \neq 0$. On the other hand, since

$$
|\Phi(1)|^{2}=1=\max _{x \in[0,1]}|\Phi(x)|^{2},
$$

we have

$$
0 \leq\left.\partial_{x}|\Phi(x)|^{2}\right|_{x=1}=\operatorname{Re} \Phi(1) \partial_{x} \operatorname{Re} \Phi(1)+\operatorname{Im} \Phi(1) \partial_{x} \operatorname{Im} \Phi(1)=\partial_{x} \operatorname{Re} \Phi(1) .
$$

Hence, one gets $\partial_{x} \Phi(1)>0$. Direct computations gives

$$
\partial_{x} u_{0}^{\prime}(1)=\partial_{x} u(1) \partial_{x} \operatorname{Re} \Phi(1)+\partial_{y} u(1) \partial_{x} \operatorname{Im} \Phi(1)=-\partial_{x} \operatorname{Re} \Phi(1)<0 .
$$

Define $u_{0}:=u_{0}^{\prime} / \partial_{x} \operatorname{Re} \Phi(1)$. We obtain $\partial_{x} u_{0}(1)=-1$ and $u_{0}\left(e^{i \theta}\right)=0$ for $|\theta| \leq \theta_{0}^{\prime}$. It remains to check that

$$
u_{0}(z) \geq c^{\prime \prime}(1-|z|),
$$

for some constant $c^{\prime \prime}>0$. Since $u_{0}(z)>0$ and $u(z)>0$ on $\mathbb{D}$ and $\partial \Omega \cap \overline{\mathbb{D}}=\left[e^{-i \theta_{0} / 2}, e^{i \theta_{0} / 2}\right]$, it is enough to check (3.10) for $z$ so that $w=\Phi(z)$ is close to $\left[e^{-i \theta_{0} / 2}, e^{i \theta_{0} / 2}\right]$. Let $w=$ $\Phi(z) \in \Omega$ close to $\left[e^{-i \theta_{0} / 2}, e^{i \theta_{0} / 2}\right]$. By our choice of $\Omega$, the axe $O w$ is transverse to $\partial \Omega$ at a unique point $w^{\prime}=\Phi\left(z^{\prime}\right)$ for $z^{\prime} \in \partial \mathbb{D}$. The $\mathcal{C}^{1}$ - boundedness of $\Phi^{-1}$ imply that $\left|w-w^{\prime}\right| \geq$ $c_{1}\left|z-z^{\prime}\right|$ for some constant $c_{1}$ independent of $\left(z, z^{\prime}\right)$. On the other hand, since $\Omega \subset \mathbb{D}$, we have $\left|w-w^{\prime}\right| \leq 1-|w|$. Hence,

$$
1-|w| \geq c_{1}\left|z-z^{\prime}\right| \geq c_{1}(1-|z|),
$$

because $z^{\prime} \in \partial \mathbb{D}$. Write $w=|w| e^{i \theta_{w}}$. Note that $\theta_{w} \in\left(\theta_{0}, \theta_{0}\right)$ if $w$ is close enough to $\left[e^{-i \theta_{0} / 2}, e^{i \theta_{0} / 2}\right]$. We deduce that

$$
u_{0}^{\prime}(z)=u(\Phi(z))=u(w) \geq c^{\prime}(1-|w|) \geq c^{\prime} c_{1}(1-|z|) .
$$

Hence, one gets (3.10). The proof is finished. 
We are now ready to introduce the Bishop equation which allows us to construct the promised family of analytic discs. Let $u_{0}$ be a function described in Lemma 3.2 and $\theta_{u_{0}}$ be the constant there. Let $\boldsymbol{\tau}_{1}, \boldsymbol{\tau}_{2} \in \overline{\mathbb{B}}_{n-1} \subset \mathbb{R}^{n-1}$. Define $\boldsymbol{\tau}_{1}^{*}:=\left(1, \boldsymbol{\tau}_{1}\right) \in \mathbb{R}^{n}$ and $\boldsymbol{\tau}_{2}^{*}:=\left(0, \boldsymbol{\tau}_{1}\right) \in \mathbb{R}^{n}$ and $\boldsymbol{\tau}:=\left(\boldsymbol{\tau}_{1}, \boldsymbol{\tau}_{2}\right)$. Let $t$ be a positive number in $(0,1)$ which plays a role as a scaling parameter in the equation (3.11) below.

In order to construct an analytic disc partly attached to $K$, it suffices to find a map

$$
U: \partial \mathbb{D} \rightarrow \mathbb{B}_{n} \subset \mathbb{R}^{n},
$$

which is Hölder continuous, satisfying the following Bishop-type equation

$$
U_{\boldsymbol{\tau}, t}(\xi)=t \boldsymbol{\tau}_{2}^{*}-\mathcal{T}_{1}\left(h\left(U_{\boldsymbol{\tau}, t}\right)\right)(\xi)-t \mathcal{T}_{1} u_{0}(\xi) \boldsymbol{\tau}_{1}^{*}
$$

Indeed, suppose that (3.11) has a solution. For simplicity, we use the same notation $U_{\boldsymbol{\tau}, t}(z)$ to denote the harmonic extension of $U_{\boldsymbol{\tau}, t}(\xi)$ to $\mathbb{D}$. Let $P_{\boldsymbol{\tau}, t}(z)$ be the harmonic extension of $h\left(U_{\boldsymbol{\tau}, t}(\xi)\right)$ to $\mathbb{D}$. Define

$$
F(z, \boldsymbol{\tau}, t):=U_{\boldsymbol{\tau}, t}(z)+i P_{\boldsymbol{\tau}, t}(z)+i t u_{0}(z) \boldsymbol{\tau}_{1}^{*}
$$

which is a family of analytic discs parametrized by $(\boldsymbol{\tau}, t)$. For any $\xi \in\left[e^{-i \theta_{u_{0}}}, e^{i \theta_{u_{0}}}\right]$, the defining formula of $F$ and the fact that $u_{0} \equiv 0$ on $\left[e^{-i \theta_{u_{0}}}, e^{i \theta_{u_{0}}}\right]$ imply that

$$
F(\xi, \boldsymbol{\tau}, t)=U_{\boldsymbol{\tau}, t}(\xi)+i P_{\boldsymbol{\tau}, t}(\xi)=U_{\boldsymbol{\tau}, t}(\xi)+i h\left(U_{\boldsymbol{\tau}, t}(\xi)\right) \in K .
$$

In other words, $F$ is $\left[e^{-i \theta_{u_{0}}}, e^{i \theta_{u_{0}}}\right]$-attached to $K$. In what follows, it is convenient to regard $U_{\boldsymbol{\tau}, t}(z)$ as a function of the variable $(z, \boldsymbol{\tau})$.

Proposition 3.3. There are a positive number $t_{1} \in(0,1)$ and a real number $c_{1}>0$ satisfying the following property. For any $t \in\left(0, t_{1}\right]$ and any $\tau \in \overline{\mathbb{B}}_{n-1}^{2}$, the equation (3.11) has a unique solution $U_{\boldsymbol{\tau}, t}$ which is $\mathcal{C}^{2, \frac{1}{2}}$ in $(\xi, \boldsymbol{\tau})$ and such that

$$
\left\|D_{(\xi, \boldsymbol{\tau})}^{j} U_{\boldsymbol{\tau}, t}\right\|_{\mathcal{C}^{\frac{1}{2}(\partial \mathbb{D})}} \leq c_{1} t
$$

for any $\boldsymbol{\tau} \in \overline{\mathbb{B}}_{n-1}^{2}$ and $j=0,1$ or 2 , where $D_{(\xi, \boldsymbol{\tau})}$ is the differential with respect to both $(\xi, \boldsymbol{\tau})$ and $D_{(\xi, \boldsymbol{\tau})}^{2}:=D_{(\xi, \boldsymbol{\tau})} \circ D_{(\xi, \boldsymbol{\tau})}$.

Proof. This is a direct consequence of a general result due to Tumanov, see [19, Th. 4.19] or see [24, Pro. 4.2] for a more simple proof adapted to our present situation.

Let $U_{\tau, t}$ be the unique solution of (3.11). As above we also use $U_{\tau, t}(z)$ to denote its harmonic extension to $\mathbb{D}$. Let $F(z, \boldsymbol{\tau}, t)$ and $P_{\boldsymbol{\tau}, t}$ be as above. Our goal is to study the behaviour of the image of the family $F(\cdot, \tau, t)$ near $K$, or in other words when $z$ is close to $\left[e^{-i \theta_{u_{0}}}, e^{i \theta_{u_{0}}}\right] \subset \partial \mathbb{D}$.

Lemma 3.4. There exists a constant $c_{2}$ so that for every $t \in\left(0, t_{1}\right]$ and every $(z, \boldsymbol{\tau}) \in$ $\overline{\mathbb{D}} \times \overline{\mathbb{B}}_{n-1}^{2}$, we have

$$
\left\|D_{(z, \boldsymbol{\tau})}^{j} U_{\boldsymbol{\tau}, t}(z)\right\| \leq c_{2} t \quad \text { and } \quad\left\|D_{(z, \boldsymbol{\tau})}^{j} P_{\boldsymbol{\tau}, t}(z)\right\| \leq c_{2} t^{2},
$$

for $j=0,1,2$.

Proof. In view of (3.5) and (3.12), the first inequality of (3.13) is obvious and for the second one, it is enough to estimate the $\mathcal{C}^{1 / 2}(\partial \mathbb{D})$-norms of $D_{(\xi, \boldsymbol{\tau})}^{j} P_{\boldsymbol{\tau}, t}(\xi)$ for $j=0,1,2$. Since $P_{\boldsymbol{\tau}, t}(\xi)=h\left(U_{\boldsymbol{\tau}, t}(\xi)\right)$ on $\partial \mathbb{D}$, we have

$$
\partial_{\xi} P_{\boldsymbol{\tau}, t}(\xi)=D h\left(U_{\boldsymbol{\tau}, t}(\xi)\right) \partial_{\xi} U_{\boldsymbol{\tau}, t}(\xi) .
$$


This combined with (3.1) and (3.12) yields that

$$
\left\|\partial_{\xi} P_{\mathbf{z}, t, \boldsymbol{\tau}}^{\prime}\right\|_{\mathcal{C}^{1 / 2}(\partial \mathbb{D})} \leq c_{0}\left\|U_{\boldsymbol{\tau}, t}\right\|_{\mathcal{C}^{1 / 2}(\partial \mathbb{D})}\left\|\partial_{\xi} U_{\boldsymbol{\tau}, t}\right\|_{\mathcal{C}^{1 / 2}(\partial \mathbb{D})} \leq c_{0} c_{1} t^{2}
$$

By similar arguments, we also have $\left|\partial_{\xi}^{j} P_{\tau}(\xi)\right| \lesssim t^{2}$ with $j=0,2$. To deal with the other partial derivatives, observe that for $0 \leq j \leq 2, D_{\tau}^{j} P_{\tau, t}$ is the harmonic extension of $D_{\boldsymbol{\tau}}^{j} h\left(U_{\boldsymbol{\tau}, t}(\cdot)\right)$ to $\mathbb{D}$. Hence, in order to estimate $D_{z}^{k} D_{\boldsymbol{\tau}}^{j} P_{\boldsymbol{\tau}, t}$ for $0 \leq k, j \leq 2$, we can apply the same reasoning as above. Thus the proof is finished.

Proposition 3.5. There are three constants $t_{2} \in\left(0, t_{1}\right], \theta_{0} \in\left(0, \theta_{u_{0}}\right)$ and $\epsilon_{0}>0$ such that for any $\boldsymbol{\tau}_{1} \in \overline{\mathbb{B}}_{n-1}$ and $t \in\left(0, t_{2}\right]$ the map $F\left(\cdot, \boldsymbol{\tau}_{1}, t\right):\left[e^{-i \theta_{0}}, e^{i \theta_{0}}\right] \times \overline{\mathbb{B}}_{n-1} \rightarrow K$ is a diffeomorphism onto its image which contains the graph of $h$ over $\mathbb{B}_{n}\left(0, t \epsilon_{0}\right)$.

Proof. By Cauchy-Riemann equations, we have

$$
\partial_{y} U_{\boldsymbol{\tau}, t}(1)=-t \partial_{x} u_{0}(1) \boldsymbol{\tau}_{1}^{*}-\partial_{x} P_{\boldsymbol{\tau}, t}(1)=t \boldsymbol{\tau}_{1}^{*}-\partial_{x} P_{\boldsymbol{\tau}, t}(1)
$$

The last term is $O\left(t^{2}\right)$ by Lemma 3.4. Thus the first component of $\partial_{y} U_{\tau, t}(1)$ is greater than $t / 2$ provided that $t \leq t_{2}$ small enough. A direct computation gives $\partial_{y} U_{\boldsymbol{\tau}, t}(1)=\partial_{\theta} U_{\boldsymbol{\tau}, t}(1)$. Consequently, the first component of $\partial_{\theta} U_{\boldsymbol{\tau}, t}(1)$ is greater than $t / 2$ for $t \leq t_{2}$.

On the other hand, by (3.11), we have $U_{\boldsymbol{\tau}, t}(1)=t \boldsymbol{\tau}_{2}^{*}$ which implies $\partial_{\boldsymbol{\tau}_{2}} U_{\boldsymbol{\tau}, t}(1)$ is a $(n, n-1)$ matrix whose the fist row is 0 and the other rows form the identity matrix. Combining with the above argument shows that $D_{\boldsymbol{\tau}_{2}, \theta} U_{\boldsymbol{\tau}, t}(1)$ is a nondegenerate matrix. This coupled with the fact that $F\left(e^{i \theta}, \boldsymbol{\tau}_{1}, t\right)=U_{\boldsymbol{\tau}, t}\left(e^{i \theta}\right)$ for $\theta \in\left[-\theta_{0}, \theta_{0}\right]$ implies the desired result. The existence of $\epsilon_{0}$ is obvious. The proof is finished.

For $a \in \mathbb{C}^{n}$ and $A \subset \mathbb{C}^{n}$, $\operatorname{dist}(a, A)$ denotes the distance from $a$ to $A$.

Proposition 3.6. There are two constants $t_{3} \in\left(0, t_{2}\right], r_{0}>0$ such that for every $t \in\left(0, t_{3}\right)$, the restriction $F_{1}$ of $F$ to $\left(\mathbb{B}_{2}\left(1, r_{0}\right) \cap \mathbb{D}\right) \times \overline{\mathbb{B}}_{n-1}^{2}$ is a diffeomorphism onto its image and for any $(z, \boldsymbol{\tau})$, we have

$$
\left|\operatorname{det} D F_{1}(z, \tau, t)\right| \gtrsim t^{2 n}[1-|z|]^{n-1}
$$

and

$$
t(1-|z|) \lesssim \operatorname{dist}\left(F_{1}(z, \tau, t), K^{\prime}\right) \lesssim t(1-|z|) .
$$

Proof. Let $r_{0}, t_{3}$ be two positive small constants to be chosen later. For the moment, we take $r_{0}$ to be small enough so that if $z=|z| e^{i \theta} \in \mathbb{B}_{2}\left(1, r_{0}\right) \cap \mathbb{D}$, then $\theta \in\left(\theta_{0}, \theta_{0}\right)$, thus we have $u_{0}\left(e^{i \theta}\right)=0$. Fix a constant $t \in\left(0, t_{3}\right]$. Provided that $t_{3}$ and $r_{0}$ are small enough we will prove in the order (3.15), (3.14) and finally that $F_{1}$ is a diffeomorphism. Extend $h$ to be a $\mathcal{C}^{3}$ function on $\mathbb{R}^{n}$. Let $\Psi: \mathbb{C}^{n} \rightarrow \mathbb{C}^{n}$ defined by

$$
\Psi(\mathbf{x}+i \mathbf{y}):=\mathbf{x}+i \mathbf{y}-i h(\mathbf{x}) .
$$

One can see without difficulty that $\Psi$ is a diffeomorphism sending $K^{\prime}$ to $\mathbb{B}_{n}$, where we embed

$$
\mathbb{R}^{n} \hookrightarrow \mathbb{R}^{n}+i \mathbb{R}^{n}=\mathbb{C}^{n}
$$

Let $F_{1}^{\prime}:=\Psi \circ F_{1}$. We have

$$
\operatorname{Im} F_{1}^{\prime}(z, \boldsymbol{\tau}, t)=P_{\boldsymbol{\tau}, t}(z)-h\left(U_{\boldsymbol{\tau}, t}(z)\right)+t u_{0}(z) \boldsymbol{\tau}_{1}^{*} \quad \text { and } \quad \operatorname{Re} F_{1}^{\prime}(z, \boldsymbol{\tau}, t)(z)=U_{\boldsymbol{\tau}, t}(z) .
$$


By the above property of $\Psi$, it suffices to prove the required property for $\left(F_{1}^{\prime}, \mathbb{B}_{n}\right)$ in place of $\left(F_{1}, K^{\prime}\right)$. Note that $P_{\boldsymbol{\tau}, t}(z)$ and $h\left(U_{\boldsymbol{\tau}, t}(z)\right)$ are identical on $\partial \mathbb{D}$. This together with (3.13) yields

$$
P_{\boldsymbol{\tau}, t}(z)-h\left(U_{\boldsymbol{\tau}, t}(z)\right)=t^{2}(1-|z|) R_{0}(z, \boldsymbol{\tau}, t),
$$

where $R_{0}(z, \boldsymbol{\tau}, t)$ is $\mathcal{C}^{1}$ in $(z, \boldsymbol{\tau})$ so that $\left\|R_{0}(\cdot, t)\right\|_{\mathcal{C}^{1}} \lesssim 1$. Remember that $t$ is fixed, so we do not consider it as a variable when taking the $\mathcal{C}^{1}$ norm. On the other hand, by our choice of $u_{0}$ and Lemma 3.2, one has $u_{0}(z) \gtrsim(1-|z|)$. By this and (3.17) and (3.16), we obtain

$$
\begin{aligned}
\operatorname{dist}\left(F_{1}(z, \boldsymbol{\tau}, t), K^{\prime}\right) & \gtrsim \operatorname{dist}\left(F_{1}^{\prime}(z, \boldsymbol{\tau}, t), \mathbb{R}^{n}\right) \\
& =\left|\operatorname{Im} F_{1}^{\prime}(z, \boldsymbol{\tau}, t)\right| \gtrsim t(1-|z|)\left|\boldsymbol{\tau}_{1}^{*}\right|-t^{2}(1-|z|) .
\end{aligned}
$$

Thus if $t$ is sufficiently small, the first inequality of (3.15) follows.

For $t_{3}$ small enough, $U_{\tau, t}(z) \in \mathbb{B}_{n}$. Hence, we get

$$
\operatorname{dist}\left(F_{1}(z, \boldsymbol{\tau}, t), K^{\prime}\right) \lesssim \operatorname{dist}\left(F_{1}^{\prime}(z, \boldsymbol{\tau}, t), \mathbb{B}_{n}\right) \lesssim\left|\operatorname{Im} F_{1}^{\prime}(z, \boldsymbol{\tau}, t)\right| .
$$

Write $z=|z| e^{i \theta} \in \mathbb{B}_{2}\left(1, r_{0}\right) \cap \mathbb{D}$. Hence $\theta \in\left[-2 r_{0}, 2 r_{0}\right] \subset\left(\theta_{0}, \theta_{0}\right)$ if $r_{0}$ is small enough. Since $u_{0}\left(e^{i \theta}\right)=0$, we deduce from (3.8) that

$$
u_{0}(z)=(1-|z|)+\theta(1-|z|) R_{1}(z)+(1-|z|)^{2} R_{2}(z)
$$

where $R_{j}$ is smooth function with $\left\|R_{j}\right\|_{\mathcal{C}^{1}} \lesssim 1$ for $j=1,2$. Put $\epsilon:=\max \left\{2 r_{0}, t\right\}$. We choose $\left(t, r_{0}\right)$ to be so small that $\epsilon<1 / 2$. Put

$$
T_{0}(z, \boldsymbol{\tau}, t):=t R_{0}(z, \boldsymbol{\tau}, t)+\left(\theta R_{1}(z)+(1-|z|) R_{2}(z)\right) \boldsymbol{\tau}_{1}^{*}
$$

which satisfies

$$
\left\|T_{0}\right\|_{\mathcal{C}^{0}} \lesssim \epsilon, \quad\left\|D_{\tau} T_{0}\right\|_{\mathcal{C}^{0}} \lesssim \epsilon
$$

because $|\theta| \leq 2 r_{0}$ and $1-|z| \leq r_{0}$. Combining (3.18), (3.17) and (3.16) gives

$$
\operatorname{Im} F_{1}^{\prime}(z, \boldsymbol{\tau}, t)=t(1-|z|)\left[\boldsymbol{\tau}_{1}^{*}+T_{0}(z, \boldsymbol{\tau}, t)\right] .
$$

Consequently, using (3.20) we obtain

$$
\left|\operatorname{Im} F_{1}^{\prime}(z, \tau, t)\right| \lesssim t(1-|z|)
$$

which proves the second inequality of (3.15).

By (3.11) and the Cauchy-Riemann equations, we have $U_{\boldsymbol{\tau}, t}(1)=t \tau_{2}^{*}$ and

$$
\partial_{y} U_{\boldsymbol{\tau}, t}(z)=-\partial_{x} P_{\boldsymbol{\tau}, t}(z)-t \partial_{x} u_{0}(z) \boldsymbol{\tau}_{1}^{*}
$$

and

$$
\partial_{x} U_{\boldsymbol{\tau}, t}(z)=\partial_{y} P_{\boldsymbol{\tau}, t}(z)+t \partial_{y} u_{0}(z) \boldsymbol{\tau}_{1}^{*}
$$

Observe that

$$
\partial_{\theta} U_{\boldsymbol{\tau}, t}\left(e^{i \theta}\right)=-\partial_{x} U_{\boldsymbol{\tau}, t}\left(e^{i \theta}\right) \sin \theta+\partial_{y} U_{\boldsymbol{\tau}, t}\left(e^{i \theta}\right) \cos \theta .
$$

These above equalities combined with (3.13) and Taylor's expansion to $U_{\boldsymbol{\tau}, t}\left(e^{i \theta}\right)$ at $\theta=0$ gives

$$
U_{\boldsymbol{\tau}, t}\left(e^{i \theta}\right)=t \boldsymbol{\tau}_{2}^{*}+t^{2} R_{3}(\theta, \boldsymbol{\tau}, t)+t \theta \boldsymbol{\tau}_{1}^{*}+t \theta^{2} R_{4}(\theta) \boldsymbol{\tau}_{1}^{*}
$$

where

$$
R_{3}(\theta, \boldsymbol{\tau}, t):=\int_{0}^{\theta}\left[\partial_{y} P_{\boldsymbol{\tau}, t}\left(e^{i s}\right) \cos s-\partial_{x} P_{\boldsymbol{\tau}, t}\left(e^{i s}\right) \sin s\right] d s
$$


which is of $\mathcal{C}^{1}$ norm $\lesssim 1$, and $R_{4}(\theta)$ is a $\mathcal{C}^{1}$ function satisfying $\left\|R_{4}\right\|_{\mathcal{C}^{1}} \lesssim 1$. Remark that in (3.22), we used the $\mathcal{C}^{3}$ differentiability of $u_{0}$ and $R_{4}$ comes from the remainder of the Taylor expansion of $u_{0}$ at 1 up to the order 2 .

Using (3.22), Taylor's expansion for $\operatorname{Re} F_{1}^{\prime}(z, \tau, t)$ at $\tilde{z}=e^{i \theta}$ implies

(3.23) $\operatorname{Re} F_{1}^{\prime}(z, \boldsymbol{\tau}, t)=t \boldsymbol{\tau}_{2}^{*}+t \theta \boldsymbol{\tau}_{1}^{*}+t^{2} R_{3}(\theta, \boldsymbol{\tau}, t)+t \theta^{2} R_{4}(\theta) \boldsymbol{\tau}_{1}^{*}+t(1-|z|) R_{5}(z, \boldsymbol{\tau}, t)$,

for some $\mathcal{C}^{1}$ function $R_{5}(z, \tau, t)$ with $\left\|R_{j}(\cdot, t)\right\|_{\mathcal{C}^{1}} \lesssim 1$. Define

$$
T_{1}(z, \boldsymbol{\tau}, t):=t R_{3}(\theta, \boldsymbol{\tau}, t)+\theta^{2} R_{4}(\theta) \boldsymbol{\tau}_{1}^{*}+(1-|z|) R_{5}(z, \boldsymbol{\tau}, t),
$$

which satisfies

$$
\left\|D_{\tau, \theta} T_{1}\right\|_{\mathcal{C}^{0}} \lesssim \epsilon
$$

where we use the polar coordinate $(|z|, \theta)$ for $z$. Combining (3.23), (3.25), (3.21) and (3.20) gives (3.14).

Let $\boldsymbol{\rho}=t \boldsymbol{\rho}_{2}+i t \boldsymbol{\rho}_{1}$ be an arbitrary point in the image of $F^{\prime}$. This means that

$$
\boldsymbol{\rho}=F_{1}^{\prime}\left(z^{0}, \boldsymbol{\tau}^{0}, t\right),
$$

for some $\left(z^{0}, \boldsymbol{\tau}^{0}\right)$. Let $\theta^{0} \in(-\pi / 2, \pi / 2)$ be the argument of $z^{0}$. Then $z^{0}=\left|z^{0}\right| e^{i \theta^{0}}$. We will prove that the equation

$$
F_{1}^{\prime}(z, \boldsymbol{\tau}, t)=\boldsymbol{\rho}
$$

has a unique solution, i.e $F_{1}^{\prime}$ is injective. The equation (3.27) is equivalent to the system of the two following equations

$$
\operatorname{Re} F_{1}^{\prime}(z, \boldsymbol{\tau}, t)=t \boldsymbol{\rho}_{2}
$$

and

$$
\operatorname{Im} F_{1}^{\prime}(z, \boldsymbol{\tau}, t)=t \boldsymbol{\rho}_{1} .
$$

Write $T_{j}=\left(T_{j 1}, \cdots, T_{j n}\right)$ for $j=0$ or 1 and $\boldsymbol{\rho}_{j}=\left(\rho_{j 1}, \cdots, \rho_{j n}\right)$ for $j=1,2$. Define

$$
\tilde{\boldsymbol{\rho}}_{1}:=\frac{\boldsymbol{\rho}_{1}}{1-|z|} .
$$

We also write $\tilde{\boldsymbol{\rho}}_{1}=\left(\tilde{\rho}_{11}, \cdots, \tilde{\rho}_{1 n}\right)$. Recall that $\boldsymbol{\tau}_{j}^{*}=\left(1, \boldsymbol{\tau}_{j}\right)$ for $j=1$ or 2 and $\boldsymbol{\tau}_{j}=$ $\left(\tau_{j 1}, \cdots, \tau_{j(n-1)}\right)$. We have

$$
\tilde{\rho}_{1 k}-\tilde{\rho}_{11} \frac{\rho_{1 k}}{\rho_{11}}=0,
$$

for $2 \leq k \leq n$. The variable $\left(\tilde{\rho}_{11}, \theta\right)$ will be used as a substitute for $z$. If $(z, \boldsymbol{\tau}, t)$ is a solution of (3.27), identifying the first component of (3.21) and (3.29) imply

$$
1+T_{01}(z, \boldsymbol{\tau}, t)=\tilde{\rho}_{11}
$$

which in turn yields $\left|\tilde{\rho}_{11}-1\right| \lesssim \epsilon$ by (3.20). Hence if $(z, \boldsymbol{\tau}, t)$ is a solution of (3.27), we get

$$
1 / 2 \leq \tilde{\rho}_{11} \leq 3 / 2 .
$$

By (3.29) again and the fact that $\tau_{1} \in \mathbb{B}_{n-1}$, one also gets

$$
\left|\frac{\rho_{1 k}}{\rho_{11}}\right| \approx\left|\tau_{1(k-1)}\right| \leq 3 / 2,
$$


for $2 \leq k \leq n$. Since $z=|z| e^{i \theta}$, we have

$$
z=\left(1-\frac{\rho_{11}}{\tilde{\rho}_{11}}\right) e^{i \theta}
$$

From now on, we will consider $T_{0}, T_{1}$ as functions of $\left(\tilde{\rho}_{11}, \theta, \boldsymbol{\tau}\right)$. Define

$$
G=\left(G_{1}, G_{2}, G_{3}\right): \overline{\mathbb{B}}_{n-1}^{2} \times\left[\frac{1}{2}, \frac{3}{2}\right] \times \mathbb{R}^{n-1} \times\left[-2 r_{0}, 2 r_{0}\right] \rightarrow \mathbb{R}^{n} \times \mathbb{R}^{n} \times \mathbb{R}^{n-1}
$$

by putting

$$
G_{1}\left(\boldsymbol{\tau}, \tilde{\boldsymbol{\rho}}_{1}, \theta\right):=\boldsymbol{\tau}_{1}^{*}+T_{0}\left(\theta, \tilde{\rho}_{11}, \boldsymbol{\tau}, t\right)-\tilde{\boldsymbol{\rho}}_{1}, \quad G_{2}\left(\boldsymbol{\tau}, \tilde{\boldsymbol{\rho}}_{1}, \theta\right):=\boldsymbol{\tau}_{2}^{*}+\theta \boldsymbol{\tau}_{1}^{*}+T_{1}\left(\theta, \tilde{\rho}_{11}, \boldsymbol{\tau}, t\right)-\boldsymbol{\rho}_{2}
$$

and

$$
G_{3}\left(\boldsymbol{\tau}, \tilde{\boldsymbol{\rho}}_{1}, \theta\right):=\left(\tilde{\rho}_{12}-\tilde{\rho}_{11} \frac{\rho_{1 k}}{\rho_{11}}, \cdots, \tilde{\rho}_{1 n}-\tilde{\rho}_{11} \frac{\rho_{1 k}}{\rho_{11}}\right) .
$$

By (3.30), (3.23) and (3.21), resolving the system (3.28)-(3.29) is equivalent to finding $\left(\boldsymbol{\tau}, \tilde{\boldsymbol{\rho}}_{1}, \theta\right)$ for which

$$
G\left(\boldsymbol{\tau}, \tilde{\boldsymbol{\rho}}_{1}, \theta\right)=0 .
$$

By (3.26), we know that $\mathbf{a}^{0}:=\left(\boldsymbol{\tau}^{0}, \tilde{\boldsymbol{\rho}}_{1}^{0}, \theta^{0}\right)$ is a solution of (3.33), where

$$
\tilde{\boldsymbol{\rho}}_{1}^{0}:=\frac{\boldsymbol{\rho}_{1}}{1-\left|z^{0}\right|} .
$$

Suppose that there is an another solution $\mathbf{a}=\left(\boldsymbol{\tau}, \tilde{\boldsymbol{\rho}}_{1}, \theta\right)$ of (3.33). By a direct computation, one gets

$$
\partial_{\tilde{\rho}_{11}}(1-|z|)=-\frac{\rho_{11}}{\tilde{\rho}_{11}^{2}}=-(1-|z|) \tilde{\rho}_{11}^{-1}=O(1-|z|) \lesssim \epsilon
$$

by (3.31). This coupled with (3.19) and (3.24) yields

$$
\left|T_{0}(\mathbf{a}, t)-T_{0}\left(\mathbf{a}^{0}, t\right)\right| \lesssim \epsilon\left|\mathbf{a}-\mathbf{a}^{0}\right|+\left|\theta-\theta^{\prime}\right|
$$

and

$$
\left|T_{1}(\mathbf{a}, t)-T_{1}\left(\mathbf{a}^{0}, t\right)\right| \lesssim \epsilon\left|\mathbf{a}-\mathbf{a}^{0}\right| .
$$

Using (3.35) and identifying the first component of the equation $G_{2}\left(\boldsymbol{\tau}, \tilde{\boldsymbol{\rho}}_{1}, \theta\right)=0$ imply

$$
\left|\theta-\theta^{0}\right| \leq\left|T_{1}(\mathbf{a}, t)-T_{1}\left(\mathbf{a}^{0}, t\right)\right| \lesssim \epsilon\left|\mathbf{a}-\mathbf{a}^{0}\right| \text {. }
$$

By doing the same thing for $G_{1}\left(\boldsymbol{\tau}, \tilde{\boldsymbol{\rho}}_{1}, \theta\right)=0$ and using (3.36), we also obtain

$$
\left|\tilde{\rho}_{11}-\tilde{\rho}_{11}^{0}\right| \leq\left|T_{0}(\mathbf{a}, t)-T_{0}\left(\mathbf{a}^{0}, t\right)\right| \lesssim \epsilon\left|\mathbf{a}-\mathbf{a}^{0}\right| .
$$

Using the last inequality, the equality $G_{3}\left(\boldsymbol{\tau}, \tilde{\boldsymbol{\rho}}_{1}, \theta\right)=0$ and (3.32), one infers

$$
\left|\tilde{\boldsymbol{\rho}}_{1}-\tilde{\boldsymbol{\rho}}_{1}^{0}\right| \lesssim\left|\tilde{\rho}_{11}-\tilde{\rho}_{11}^{0}\right| \lesssim \epsilon\left|\mathbf{a}-\mathbf{a}^{0}\right|
$$

Similarly, using $G_{1}\left(\boldsymbol{\tau}, \tilde{\boldsymbol{\rho}}_{1}, \theta\right)=0$ gives

$$
\left|\boldsymbol{\tau}_{1}-\boldsymbol{\tau}_{1}^{0}\right| \leq\left|T_{0}(\mathbf{a}, t)-T_{0}\left(\mathbf{a}^{0}, t\right)\right|+\left|\tilde{\boldsymbol{\rho}}_{1}-\tilde{\boldsymbol{\rho}}_{1}^{0}\right| \lesssim \epsilon\left|\mathbf{a}-\mathbf{a}^{0}\right| .
$$

Finally, using $G_{2}\left(\boldsymbol{\tau}, \tilde{\boldsymbol{\rho}}_{1}, \theta\right)=0$ gives

$$
\left|\boldsymbol{\tau}_{2}-\boldsymbol{\tau}_{2}^{0}\right| \lesssim \epsilon\left|\mathbf{a}-\mathbf{a}^{0}\right|
$$

Summing the inequalities from (3.36) to (3.39) and taking into account that

$$
\left|\mathbf{a}-\mathbf{a}^{0}\right| \leq\left|\boldsymbol{\tau}_{2}-\boldsymbol{\tau}_{2}^{0}\right|+\left|\boldsymbol{\tau}_{1}-\boldsymbol{\tau}_{1}^{0}\right|+\left|\tilde{\boldsymbol{\rho}}_{1}-\tilde{\boldsymbol{\rho}}_{1}^{0}\right|+\left|\theta-\theta^{0}\right|
$$


show that $\mathbf{a}=\mathbf{a}^{0}$. This means that (3.33) has a unique solution, or equivalently, so does (3.27) if $r_{0}$ and $t$ are small enough. The proof is finished.

Let $\Omega$ be a simply connected subdomain of $\mathbb{D}$ with smooth boundary such that $\Omega$ is strictly convex and $\bar{\Omega} \cap \overline{\mathbb{D}}=\left[e^{-i \theta_{1}}, e^{i \theta_{1}}\right]$ for some $\theta_{1} \in\left(0, \theta_{0}\right)$ and $\bar{\Omega} \subset \mathbb{B}_{2}\left(1, r_{0}\right)$. By Painvelé's theorem as in the proof of Lemma 3.2, there is a smooth diffeomorphism $\Phi$ from $\overline{\mathbb{D}}$ to $\bar{\Omega}$ which is a biholomorphism from $\mathbb{D}$ to $\Omega$ and $\Phi(1)=1$.

Define $\tilde{F}(z, \boldsymbol{\tau}, t):=F(\Phi(z), \boldsymbol{\tau}, t)$ which is again a $\mathcal{C}^{2,1 / 2}$ family of analytic discs partly attached to $K$.

Proposition 3.7. ( $i$ ) There are positive constants $\tilde{\theta}_{0}$ and $\tilde{\epsilon}_{0}$ so that for every $\tau_{1} \in \overline{\mathbb{B}}_{n-1}$ and $t \in\left(0, t_{3}\right]$, the restriction map $\tilde{F}\left(\cdot, \boldsymbol{\tau}_{1}, t\right):\left[e^{-i \tilde{\theta}_{0}}, e^{i \tilde{\theta}_{0}}\right] \times \overline{\mathbb{B}}_{n-1} \rightarrow K^{\prime}$ is a diffeomorphism onto its image which contains the graph of $h$ over $\mathbb{B}_{n}\left(0, t \tilde{\epsilon}_{0}\right)$.

(ii) Let $t_{3}$ be the constant in Proposition 3.6. Then for any $t \in\left(0, t_{3}\right]$, the map $\tilde{F}(\cdot, t)$ is a diffeomorphism from $\mathbb{D} \times \overline{\mathbb{B}}_{n-1}^{2}$ onto its image in $\mathbb{D}^{n} \subset \mathbb{C}^{n}$, and for any $(z, \boldsymbol{\tau})$ we have

$$
|\operatorname{det} D \tilde{F}(z, \boldsymbol{\tau}, t)| \gtrsim t^{n+1} \operatorname{dist}^{n-1}\left(\tilde{F}(z, \boldsymbol{\tau}, t), K^{\prime}\right)
$$

and

$$
t(1-|z|) \lesssim \operatorname{dist}\left(\tilde{F}(z, \tau, t), K^{\prime}\right)
$$

Proof. Property $(i)$ is a direct consequence of Propositions 3.5. By the differentiability of $\Phi^{-1}$ on $\bar{\Omega}$, we have $(1-|\Phi(z)|) \gtrsim 1-|z|$ for every $z \in \mathbb{D}$. Hence, by (3.15), we get (3.41). The inequality (3.40) follows immediately from the fact that

$$
\left|\operatorname{det} D F_{1}(z, \boldsymbol{\tau}, t)\right| \gtrsim t^{n+1} \operatorname{dist}^{n-1}\left(F_{1}(z, \boldsymbol{\tau}, t), K^{\prime}\right)
$$

which is in turn implied by (3.15) and (3.14). The proof is finished.

Using the local coordinates of $K$ at the beginning of this section and choosing $t=t_{3}$, the last proposition can be rephrased as follows.

Proposition 3.8. Let a be an arbitrary point of $K$. Then there exist positive constants $\tilde{\epsilon}_{a}, \tilde{\theta}_{a}$ and $a \mathcal{C}^{2,1 / 2}$ diffeomorphism $\tilde{F}_{a}: \mathbb{D} \times \overline{\mathbb{B}}_{n-1}^{2} \rightarrow X$ onto its image such that the two following properties hold:

(i) for every $\boldsymbol{\tau}_{1} \in \overline{\mathbb{B}}_{n-1}$, the restriction map $\tilde{F}_{a}\left(\cdot, \boldsymbol{\tau}_{1}\right):\left[e^{-i \tilde{\theta}_{a}}, e^{i \tilde{\theta}_{a}}\right] \times \mathbb{B}_{n-1} \rightarrow K$ is a diffeomorphism onto its image which contains the graph of $h$ over $\mathbb{B}_{K}\left(a, \tilde{\epsilon}_{a}\right)$.

(ii) there is an open relatively compact neighborhood $K_{a}^{\prime}$ of a in $K$ such that for any $(z, \tau)$, we have

$$
\left|\operatorname{det} D \tilde{F}_{a}(z, \boldsymbol{\tau})\right| \gtrsim \operatorname{dist}^{n-1}\left(\tilde{F}_{a}(z, \boldsymbol{\tau}), K_{a}^{\prime}\right)
$$

and

$$
(1-|z|) \lesssim \operatorname{dist}\left(\tilde{F}_{a}(z, \boldsymbol{\tau}), K_{a}^{\prime}\right)
$$

\section{SOME ESTIMATES FOR P.S.H. FUNCTIONS}

In this section, we will prove some key estimates for p.s.h functions and their $d d^{c}$ on $\mathbb{C}^{n}$. For a Borel subset $A$ of $\mathbb{R}^{m}$ with $m \in \mathbb{N}$, denote by $|A|$ the volume of $A$ with respect to the canonical volume form $\operatorname{vol}_{\mathbb{R}^{m}}$. In what follows, for simplicity, we will write $\int_{A} f$ instead of $\int_{A} f d v o l_{\mathbb{R}^{m}}$ for every Borel set $A \subset \mathbb{R}^{m}$ and every integrable function $f$ on $A$. In particular, this convention is applied to $\mathbb{C}^{n}=\mathbb{R}^{2 n}$. 
Lemma 4.1. Let $V$ be an open subset of $\mathbb{C}^{n}$ and let $V_{1}$ be a compact subset of $V$. Let $\varphi$ be a p.s.h. function on $V$. Then there exists a constant $c$ independent of $\varphi$ such that for any Borel set $V_{2} \subset V_{1}$, we have

$$
\int_{V_{2}}|\varphi| \leq c\left|V_{2}\right| \max \left\{1,-\log \left|V_{2}\right|\right\} \int_{V}|\varphi|
$$

Proof. If $\varphi \equiv 0$ or $\int_{V}|\varphi|=\infty$, then there is nothing to prove. Now suppose $\varphi \neq 0$ and $\int_{V}|\varphi|<\infty$. Let $\varphi_{1}=\varphi / \int_{V}|\varphi|$. We have $\int_{V}\left|\varphi_{1}\right|=1$. As a result, there exist two positive constants $\left(c_{1}, \alpha_{1}\right)$ independent of $\varphi_{1}$ for which

$$
\int_{V_{1}} e^{\alpha_{1}\left|\varphi_{1}\right|} \leq c_{1}
$$

Let $\mathbf{1}_{V_{2}}$ be the characteristic function of $V_{2}$. Let $\mu:=\left|V_{2}\right|^{-1} \mathbf{1}_{V_{2}} v l_{\mathbb{C}^{n}}$ which is a probability measure supported in $V_{2}$. We have

$$
\int_{V_{2}}\left|\varphi_{1}\right|=\alpha_{1}^{-1} \int_{V_{2}} \log e^{\alpha_{1}\left|\varphi_{1}\right|}=\alpha_{1}^{-1}\left|V_{2}\right| \int_{V_{2}} \log e^{\alpha_{1}\left|\varphi_{1}\right|} d \mu .
$$

This together with the concavity of the log function implies

$$
\int_{V_{2}}\left|\varphi_{1}\right| \leq \alpha_{1}^{-1}\left|V_{2}\right| \log \int_{V_{2}} e^{\alpha_{1}\left|\varphi_{1}\right|} d \mu
$$

which, by (4.2), is less than or equal to

$$
\alpha_{1}^{-1}\left|V_{2}\right|\left(\log c_{1}-\log \left|V_{2}\right|\right) .
$$

Hence (4.1) follows. The proof is finished.

Now let $h, K^{\prime}$ be as in Section 3. Let $\epsilon$ be a real positive number and $K_{\epsilon}^{\prime}$ the compact subset of $\mathbb{C}^{n}$ consisting of points of distance $\leq \epsilon$ to $K^{\prime}$. Obviously, the volume of $K_{\epsilon}^{\prime}$ is $\lesssim \epsilon^{n}$. Using Lemma 4.1 for $V_{2}=K_{\epsilon}^{\prime}$, we get the following.

Corollary 4.2. Let $V$ be an open subset of $\mathbb{C}^{n}$ containing $H_{1}$. Let $\varphi$ be a p.s.h. function on $V$. Then there is a constant $c$ independent of $\varphi$ for which

$$
\int_{K_{\epsilon}^{\prime}}|\varphi| \leq c \epsilon^{n}|\log \epsilon| \int_{V}|\varphi|
$$

for every $\epsilon \leq 1 / 2$.

Now we will give a similar estimate for the mass of $d d^{c} \varphi$ on $K_{\epsilon}^{\prime}$. We begin with a general result.

Lemma 4.3. Let $V, V_{1}, V_{2}$ be open subsets of $\mathbb{C}^{n}$ such that $V_{2} \Subset V_{1} \Subset V$. Let $T$ be a closed positive current of bidimension $(p, p)$ on $V$ and $\lambda$ a real number $>1$. Let $\varphi$ and $\rho$ be two bounded p.s.h. functions on $V$. Let $A$ be a subset of $V_{2}$ and $a_{\epsilon, \varphi}$ be an upper bound for $|\varphi|$ on $V_{1} \cap\{\rho \leq \epsilon\}$ for $\epsilon>0$. Assume that $\rho$ is bounded by 1 on $V$. Then there is a constant $c$ independent of $T, A, \rho, \varphi$ such that

$$
\int_{A \cap\{\rho \leq \epsilon\}} T \wedge\left(d d^{c} \varphi\right)^{p} \leq c\left[\epsilon^{-1} a_{\lambda \epsilon, \varphi}\right]^{p}\|T\|_{V_{1}},
$$

for every $\epsilon \in(0,1)$. 
Proof. We prove (4.4) by induction on $p$. When $p=0$, the conclusion is obvious. Suppose (4.4) holds for $p-1$. We need to prove its validity for $p$. Let $\chi$ be a smooth function compactly supported in some $V_{1}^{\prime} \Subset V_{1}$ such that $0 \leq \chi \leq 1$ and $\chi \equiv 1$ on $V_{2}$. Let $\epsilon$ be a positive constant. Choose a constant $\lambda^{\prime} \in(1, \lambda)$. Define

$$
\rho_{\epsilon}:=\max \left\{\rho, \lambda^{\prime} \epsilon\right\}-\max \{\rho, \epsilon\}
$$

which is the difference of two bounded p.s.h. functions on $V$. Clearly, we have $0 \leq \rho_{\epsilon} \leq$ $\left(\lambda^{\prime}-1\right) \epsilon$ and $\rho_{\epsilon}=\left(\lambda^{\prime}-1\right) \epsilon$ on $\{\rho \leq \epsilon\}$ and $\rho_{\epsilon}=0$ on $\left\{\rho \geq \lambda^{\prime} \epsilon\right\}$. This yields

$$
\int_{A \cap\{\rho \leq \epsilon\}} T \wedge\left(d d^{c} \varphi\right)^{p} \leq \frac{\epsilon^{-1}}{\lambda^{\prime}-1} \int_{V} \chi \rho_{\epsilon} T \wedge\left(d d^{c} \varphi\right)^{p} \lesssim \epsilon^{-1} \int_{V} \chi \rho_{\epsilon} T \wedge\left(d d^{c} \varphi\right)^{p}
$$

which is, by integration by parts, equal to

$$
\epsilon^{-1} \int_{V} \rho_{\epsilon} \varphi d d^{c} \chi \wedge T \wedge\left(d d^{c} \varphi\right)^{p-1}+\epsilon^{-1} \int_{V} \varphi \chi d d^{c} \rho_{\epsilon} \wedge T \wedge\left(d d^{c} \varphi\right)^{p-1}+R
$$

where

$$
R=2 \epsilon^{-1} \int_{V} \varphi d \chi \wedge d^{c} \rho_{\epsilon} \wedge T \wedge\left(d d^{c} \varphi\right)^{p-1} .
$$

Denote by $R_{1}$ and $R_{2}$ respectively the first and second terms in (4.6). We are now going to estimate $R_{1}, R$ and finally $R_{2}$. Let $\omega$ be the canonical Kähler form on $\mathbb{C}^{n}$. Since $d d^{c} \chi \lesssim \omega$ and $|\varphi| \leq a_{2 \epsilon, \varphi}$ on $\operatorname{supp} \rho_{\epsilon}$, we get

$$
R_{1} \leq \epsilon^{-1} a_{\lambda^{\prime} \epsilon, \varphi} \int_{V_{1}^{\prime} \cap\left\{\rho \leq \lambda^{\prime} \epsilon\right\}} \omega \wedge T \wedge\left(d d^{c} \varphi\right)^{p-1} .
$$

Applying the induction hypothesis to $\omega \wedge T, \lambda^{\prime} \epsilon$ in place of $T, \epsilon$ implies

$$
R_{1} \leq \epsilon^{-1} a_{\lambda^{\prime} \epsilon, \varphi} \int_{V_{1}^{\prime} \cap\left\{\rho \leq \lambda^{\prime} \epsilon\right\}} \omega \wedge T \wedge\left(d d^{c} \varphi\right)^{p-1} \lesssim\left[\epsilon^{-1} a_{\lambda \epsilon, \varphi}\right]^{p} .
$$

As to $R$, the Cauchy-Schwarz inequality applied to a suitable scalar product gives

$$
\begin{aligned}
|R|^{2} & \leq \epsilon^{-2} \int_{V_{1}^{\prime}}|\varphi| \mathbf{1}_{\left\{\rho \leq \lambda^{\prime} \epsilon\right\}} d \chi \wedge d^{c} \chi \wedge T \wedge\left(d d^{c} \varphi\right)^{p-1} \int_{V_{1}^{\prime}}|\varphi| d \rho_{\epsilon} \wedge d^{c} \rho_{\epsilon} \wedge T \wedge\left(d d^{c} \varphi\right)^{p-1} \\
& \lesssim\left[\epsilon^{-1} a_{\lambda^{\prime} \epsilon, \varphi}\right]^{p+1} \int_{V_{1}^{\prime}} d \rho_{\epsilon} \wedge d^{c} \rho_{\epsilon} \wedge T \wedge\left(d d^{c} \varphi\right)^{p-1}
\end{aligned}
$$

by induction hypothesis and the fact that $d \rho_{\epsilon} \wedge d^{c} \rho_{\epsilon}$ is positive and supported on $\left\{\rho \leq \lambda^{\prime} \epsilon\right\}$. Denote by $R^{\prime}$ the last integral. Since $\rho_{\epsilon}$ is the difference of two bounded p.s.h. functions on $V$, so is $\rho_{\epsilon}^{2}$. More precisely, since $|\rho| \leq 1$ on $V$ we can find four p.s.h function $\psi_{j}$ with $1 \leq j \leq 4$ so that they are bounded independent of $\epsilon$ and

$$
\rho_{\epsilon}^{2}=\psi_{1}-\psi_{2} \quad \text { and } \quad \rho_{\epsilon}=\psi_{3}-\psi_{4} .
$$

We also have

$$
d d^{c} \rho_{\epsilon}^{2}=2 d \rho_{\epsilon} \wedge d^{c} \rho_{\epsilon}+2 \rho_{\epsilon} d d^{c} \rho_{\epsilon} .
$$

Note that each side of the last equality is well-defined. Substituting this to the defining formula of $R^{\prime}$, then using (4.9), one gets

$$
R^{\prime} \leq \sum_{j=1}^{4} \int_{V_{1}^{\prime} \cap\left\{\rho \leq \lambda^{\prime} \epsilon\right\}} d d^{c} \psi_{j} \wedge T \wedge\left(d d^{c} \varphi\right)^{p-1}
$$


which, by induction hypothesis, is $\lesssim$

$$
\left[\epsilon^{-1} a_{\lambda \epsilon, \varphi}\right]^{p-1} \sum_{j=1}^{4}\left\|d d^{c} \psi_{j} \wedge T\right\|_{V_{1}^{\prime \prime}}
$$

where $V_{1}^{\prime \prime}$ be a relatively compact subset of $V_{1}$ which is open and contains $\bar{V}_{1}^{\prime}$. By the Chern-Levine-Nirenberg inequality, the last sum is $\lesssim\|T\|_{V_{1}}$. Combining with (4.8), we obtain

$$
R \leq\left[\epsilon^{-1} a_{\lambda \epsilon, \varphi}\right]^{p}\|T\|_{V_{1}} .
$$

Bounding $R_{2}$ is done similarly. The proof is finished.

Lemma 4.4. Let $f$ be a real $\mathcal{C}^{2}$ function on an open set $V \subset \mathbb{C}^{n}$. Let $g(t):=|t| \log (|t|+2)$ for $t \in \mathbb{R}$. Let $\omega$ be the canonical Kähler form on $\mathbb{C}^{n}$. Then we have

$$
12 d d^{c}(g \circ f) \geq d f \wedge d^{c} f-2 n\left\|D^{2} f\right\|_{L^{\infty}(V)} \omega
$$

as currents on $V$.

Proof. By direct computations, one obtains for $t>0$,

$$
g^{\prime}(t)=1-\frac{2}{2+t}+\log (2+t), \quad g^{\prime \prime}(t)=\frac{2}{(2+t)^{2}}+\frac{1}{2+t}
$$

and for $t<0$,

$$
g^{\prime}(t)=-1+\frac{2}{2-t}-\log (2-t), \quad g^{\prime \prime}(t)=\frac{2}{(2-t)^{2}}+\frac{1}{2-t} .
$$

For $k \geq 3$, we are going to construct a sequence of $\mathcal{C}^{2}$ convex function $g_{k}$ of uniformly bounded $L^{\infty}$ norm converging pointwise to $g$. To this end, we define

$$
q_{k}(t):=\frac{2}{(2+|t|)^{2}}+\frac{1}{2+|t|} \text { for } t \geq 1 / k
$$

and on $[-1 / k, 1 / k]$, let $q_{k}(t)$ be the piece-wise affine function satisfying the two following properties:

(i) $q_{k}$ is affine on $[-1 / k, 0]$ and on $[0,1 / k], q_{k}(0)=2 k g^{\prime}(1 / k)-q_{k}(1 / k) \geq 1$,

(ii) $q_{k}$ is continuous on $\mathbb{R}$.

The value of $q(0)$ is in fact chosen such that

$$
\int_{-1 / k}^{1 / k} q_{k}(t) d t=g^{\prime}(1 / k)-g^{\prime}(-1 / k) .
$$

This property ensures the existence of a unique $\mathcal{C}^{2}$ convex function $g_{k}(t)$ on $\mathbb{R}$ satisfying $g_{k}(t) \equiv g(t)$ for $|t| \geq 1 / k$ and $g_{k}^{\prime \prime}(t)=q_{k}(t)$. One can check that $g_{k}$ is uniformly bounded and $g_{k}$ converges to $g$. Hence $g_{k}(f)$ converges weakly to $g(f)$ as currents. On the other hand, direct computations give

$$
g_{k}^{\prime \prime}(f) \geq \min \{1 / 3,2 k \log 2-1\}=1 / 3, \quad\left|g_{k}^{\prime}(t)\right| \leq \log 3+2 \leq 4
$$

for $|t| \leq 1$ and

$$
d d^{c} g_{k}(f)=g_{k}^{\prime \prime}(f) d f \wedge d^{c} f+g_{k}^{\prime}(f) d d^{c} f \geq 12^{-1}\left(d f \wedge d^{c} f-2 n\left\|D^{2} f\right\|_{L^{\infty}} \omega\right) .
$$

The proof is finished. 
Proposition 4.5. Let $\varphi$ be a p.s.h. function on an open set $V \subset \mathbb{C}^{n}$. Let $A$ be a generic $\mathcal{C}^{3}$ submanifold of dimension $n$ of $V$. Let $A_{1}$ be a compact subset of $A$ and for $\epsilon>0$, let $A_{1, \epsilon}$ be the set of points in $\mathbb{C}^{n}$ of distance $\leq \epsilon$ to $A_{1}$. Then there is a constant $c$ independent of $\varphi, \epsilon$ for which we have

$$
\int_{A_{1, \epsilon}} d d^{c} \varphi \wedge \omega^{n-1} \leq c \epsilon^{n-1} \int_{V}|\varphi|
$$

where $\omega$ is the canonical Kähler form of $\mathbb{C}^{n}$.

Proof. Let $\delta$ be a small positive number which will be chosen later. Observe that the question is local. By using a partition of unity and Lemma 3.1, it is enough to prove the desired result for the case where $A$ is the graph of a $\mathcal{C}^{3}$ map $h$ over $\mathbb{B}_{n}(0,3 \delta)$ such $h(0)=D h(0)=D^{2} h(0)=0$ and $\|h\|_{\mathcal{C}^{3}}$ is bounded independently of chosen local charts (hence, in particular, independent of $\delta$ ); and $A_{1}$ is the part of the graph over $\overline{\mathbb{B}}_{n}(0, \delta)$. We can assume that

$$
A_{1, \epsilon}=\left\{\mathbf{x}+i \mathbf{y}: \mathbf{x} \in \overline{\mathbb{B}}_{n}(0, \delta),|\mathbf{y}-h(\mathbf{x})| \leq \epsilon\right\}
$$

and $V=\mathbb{B}_{n}(0,3 \delta)+i \mathbb{B}_{n}$.

Let $g$ be the function defined in Lemma 4.4. We write $\mathbf{z}=\left(z_{1}, \cdots, z_{n}\right), \mathbf{y}=\left(y_{1}, \cdots, y_{n}\right)$ and $h=\left(h_{1}, \cdots, h_{n}\right)$. Since $\left|D^{2} h\right| \lesssim \delta$ on $\mathbb{B}_{n}(0,3 \delta)$, one has

$$
\mid D^{2}\left(y_{j}-h_{j}(\mathbf{x})\right) \| \lesssim \delta
$$

for $1 \leq j \leq n$. Using this and Lemma 4.4, we see that the function

$$
\rho(\mathbf{z}):=\sum_{j=1}^{n} g\left(y_{j}-h_{j}(\mathbf{x})\right)
$$

satisfies

$$
d d^{c} \rho \geq \sum_{j=1}^{n}\left(\frac{i}{4 \pi} d z_{j} \wedge d \bar{z}_{j}-\delta M d z_{j} \wedge d \bar{z}_{j}\right),
$$

for some constant $M$ independent of $\delta$. Thus if $\delta$ is small enough independently of $\epsilon$, the function $\rho$ is p.s.h. on $V$. It is clear that $A_{1, \epsilon} \subset A_{1} \cap\{\rho \leq 2 n \epsilon\}$. Let $\varphi_{1}(\mathbf{z}):=|\mathbf{y}-h(\mathbf{x})|^{2}$. A direct computation shows that $\varphi_{1}$ is also p.s.h. on $V$. Note that $\left|\varphi_{1}\right| \lesssim \epsilon^{2}$ on $\{\rho \leq 2 \epsilon$. $\}$ Now applying Lemma 4.3 to $\left(\rho, \varphi_{1}\right)$ and to $T:=d d^{c} \varphi$, we obtain

$$
\int_{A_{1, \epsilon}} d d^{c} \varphi \wedge\left(d d^{c} \varphi_{1}\right)^{n-1} \lesssim \epsilon^{n-1}\left\|d d^{c} \varphi\right\|_{\mathbb{B}_{n}(0,2 \delta)+i \mathbb{B}_{n}(0,1 / 2)} \lesssim \epsilon^{n-1} \int_{V}|\varphi| .
$$

The last inequality together with the fact that $d d^{c} \varphi_{1} \gtrsim \omega$ gives the desired result. The proof is finished.

Note that a similar technique was used by Sibony in [21] when dealing with the extension of positive closed currents (or more generally pluripositive currents) through a CR submanifold. For $\epsilon \in(0,1]$, let $K_{\epsilon}^{\prime}$ be as above. The following is just a direct consequence of Proposition 4.5 .

Corollary 4.6. Let $V$ be an open subset of $\mathbb{C}^{n}$ containing $K_{1}^{\prime}$. Let $\varphi$ be a p.s.h. function on $V$. Then there is a constant $c$ independent of $\varphi, \epsilon$ for which we have

$$
\int_{K_{\epsilon}^{\prime}} d d^{c} \varphi \wedge \omega^{n-1} \leq c \epsilon^{n-1} \int_{V}|\varphi|
$$


where $\omega$ is the canonical Kähler form of $\mathbb{C}^{n}$.

Now we are going to give some applications of these above estimates to our present problem. Firstly, we prove some auxiliary lemmas. Let $t_{3}, \tilde{\epsilon}_{0}$ and $\tilde{\theta}_{0}$ be the constants in Proposition 3.7. Let $\tilde{F}$ be the family of analytic discs defined there. For simplicity, from now on, we denote $\tilde{F}\left(z, \boldsymbol{\tau}, t_{3}\right)$ by $\tilde{F}(z, \boldsymbol{\tau})$. Recall that the image of $\tilde{F}$ is contained in $\mathbb{D}^{n}$. Put $\tilde{\epsilon}_{0}^{\prime}:=t_{3} \tilde{\epsilon}_{0}$.

Lemma 4.7. There exists a positive constant $c_{0}$ such that for any Borel function $g$ on $\mathbb{D}^{n}$, we have

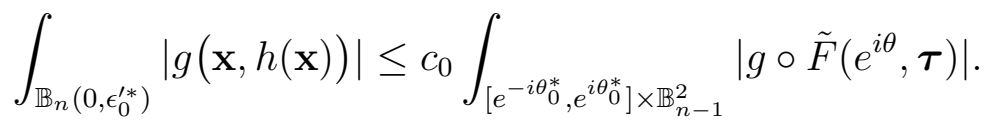

Proof. This is a direct consequence of Property $(i)$ of Proposition 3.7 and the change of variables theorem. The proof is finished.

Lemma 4.8. Let $g$ be a Borel function on $\mathbb{D}^{n}$.

(i) If $n=1$, then

$$
\int_{\mathbb{D} \times \mathbb{B}_{n-1}(0,1)^{2}}|g \circ \tilde{F}(z, \boldsymbol{\tau})| \leq c_{1} \int_{\mathbb{D}^{n}}|g(\mathbf{z})|
$$

for some constant $c_{1}$ independent of $g$.

(ii) Assume $n>1$. Let $t_{0}$ and $\delta_{0}$ be two positive real numbers such that $t_{0}+\delta_{0}>n-1>\delta_{0}$. Let

$$
M_{g}:=\sup _{\epsilon \in(0,1)} \epsilon^{-t_{0}} \int_{K_{\epsilon}^{\prime}}|g(\mathbf{z})|
$$

and $\lambda_{0}:=t_{0}+\delta_{0}-n+1$. Assume $M_{g}<\infty$. Then we have

$$
\int_{\mathbb{D} \times \mathbb{B}_{n-1}^{2}}(1-|z|)^{\delta_{0}}|g \circ \tilde{F}(z, \boldsymbol{\tau})| \leq \frac{2^{t_{0}} c_{1} M_{g}}{\lambda_{0}}\left[\int_{\mathbb{D}^{n}}|g(\mathbf{z})|\right]^{\frac{\lambda_{0}}{t_{0}}},
$$

for some constant $c_{1}$ independent of $g, t_{0}, \delta_{0}$.

Proof. When $n=1$, the desired inequality is a direct application of the change of variables theorem and (3.40). Consider now $n>1$. Put $Y:=\mathbb{D} \times \mathbb{B}_{n-1}^{2}$. Let $\epsilon$ be a small positive number which will be chosen later. Set

$$
Y_{\epsilon, 0}:=\left\{(z, \boldsymbol{\tau}) \in Y: \operatorname{dist}\left(\tilde{F}(z, \boldsymbol{\tau}), K^{\prime}\right) \geq \epsilon\right\}
$$

and

$$
Y_{\epsilon, k}:=\left\{(z, \boldsymbol{\tau}) \in Y: 2^{-k} \epsilon \leq \operatorname{dist}\left(\tilde{F}(z, \boldsymbol{\tau}), K^{\prime}\right) \leq 2^{-k+1} \epsilon\right\},
$$

for $k \in \mathbb{N}$. It is clear that $Y=\cup_{k=0}^{\infty} Y_{\epsilon, k}$. By definition of $K_{\epsilon}^{\prime}$, we have

$$
\tilde{F}\left(Y_{\epsilon, k}\right) \subset H_{2^{-k+1}}{ } .
$$


Denote by $\operatorname{vol}_{Y}$ the canonical volume form on $Y$. Write

$$
\begin{aligned}
\int_{Y}(1-|z|)^{\delta_{0}}|g \circ \tilde{F}| d v o l_{Y} & =\sum_{k=0}^{\infty} \int_{Y_{\epsilon, k}}(1-|z|)^{\delta_{0}}|g \circ \tilde{F}| d v o l_{Y} \\
& \lesssim \sum_{k=0}^{\infty} \int_{Y_{\epsilon, k}}(1-|z|)^{\delta_{0}}|g \circ \tilde{F}(z, \boldsymbol{\tau})| \frac{|\operatorname{det} D \tilde{F}(z, \boldsymbol{\tau})|}{\operatorname{dist}^{n-1}\left(\tilde{F}(z, \boldsymbol{\tau}), K^{\prime}\right)} d v o l_{Y}
\end{aligned}
$$

(by (3.40) )

$$
\lesssim \sum_{k=0}^{\infty}\left(2^{-k} \epsilon\right)^{-n+1+\delta_{0}} \int_{Y_{\epsilon, k}}|g \circ \tilde{F}||\operatorname{det} D \tilde{F}| d v o l_{Y}
$$

by definition of $Y_{\epsilon, k}$, (3.41) and the fact that $-n+1+\delta_{0}<0$. By change of variables, the last integral equals

$$
\int_{\tilde{F}\left(Y_{\epsilon, k}\right)}|g|
$$

which is, for $k \geq 1$, less than or equal to

$$
\int_{H_{2-k+1} \epsilon}|g| \leq\left(2^{-k+1} \epsilon\right)^{t_{0}} M_{g}
$$

by definition of $M_{g}$ and (4.14). This coupled with (4.15) yields that

$$
\begin{aligned}
\int_{Y}(1-|z|)^{\delta_{0}}|g \circ \tilde{F}| & \lesssim \epsilon^{-n+1+\delta_{0}} \int_{\mathbb{D}^{n}}|g|+2^{t_{0}} \epsilon^{\lambda_{0}} M_{g} \sum_{k=1}^{\infty} 2^{-k \lambda_{0}} \\
& \lesssim \epsilon^{-n+1+\delta_{0}} \int_{\mathbb{D}^{n}}|g|+\frac{2^{t_{0}} \epsilon^{\lambda_{0}} M_{g}}{2^{\lambda_{0}}-1}
\end{aligned}
$$

Choose $\epsilon=\|g\|_{L^{1}\left(\mathbb{D}^{n}\right)}^{1 / t_{0}}$. Using (4.16) and the fact that $2^{\lambda_{0}} \geq 1+\lambda_{0}$, we get the desired inequality. The proof is finished.

The following will be crucial for our later purpose.

Corollary 4.9. Let $V$ be an open subset of $\mathbb{C}^{n}$ containing $K_{1}^{\prime} \cup \overline{\mathbb{D}}^{n}$. Let $\varphi$ be a p.s.h. function on $V$. Let $\delta \in(0,1)$. Define $\gamma:=\delta /(n-1)$ if $n>1$ and $\gamma=1$ otherwise. Then we have

$$
\int_{\mathbb{D} \times \mathbb{B}_{n-1}^{2}}(1-|z|)^{\delta} d d^{c}(\varphi \circ \tilde{F})(z, \boldsymbol{\tau}) \lesssim_{\delta}\|\varphi\|_{L^{1}(V)}^{\gamma} .
$$

Furthermore, we have

$$
\int_{\{1-2 \epsilon \leq|z| \leq 1\} \times \mathbb{B}_{n-1}^{2}}(1-|z|) d d^{c}(\varphi \circ \tilde{F})(z, \boldsymbol{\tau}) \lesssim_{\delta} \epsilon^{1-\frac{\delta(n-1)}{\delta+n-1}} \max \left\{\|\varphi\|_{L^{1}(V)}^{\gamma},\|\varphi\|_{L^{1}(V)}\right\}
$$

for every $\epsilon \in(0,1)$.

Proof. Firstly we prove (4.17). The case where $n=1$ is clear. Consider $n>1$. Let $V_{1} \Subset V$ be an open subset of $V$. Fix a decreasing sequence of smooth p.s.h. functions $\varphi_{l}$ converging pointwise to $\varphi$ on $V_{1}$ and $\left\|\varphi_{l}\right\|_{L^{1}\left(V_{1}\right)} \leq 2\|\varphi\|_{L^{1}(V)}$. Let $\delta \in(0,1)$. Since

$$
d d^{c} \varphi_{l}=\frac{i}{\pi} \sum_{1 \leq j, k \leq n} \frac{\partial^{2} \varphi_{l}}{\partial z_{j} \partial \bar{z}_{k}} d z_{j} \wedge d \bar{z}_{k} \geq 0
$$


using Corollary 4.6, there is a positive constant $c$ independent of $\varphi$ such that for every $j, k, l$ we have

$$
\int_{K_{\epsilon}^{\prime}}\left|\frac{\partial^{2} \varphi_{l}}{\partial z_{j} \partial \bar{z}_{k}}\right| \leq c \epsilon^{n-1} \int_{V_{1}}\left|\varphi_{l}\right| \leq c \epsilon^{n-1}\|\varphi\|_{L^{1}(V)}
$$

which infers that the constant $M_{g}$, defined in Lemma 4.8 for

$$
g:=\frac{\partial^{2} \varphi_{l}}{\partial z_{j} \partial \bar{z}_{k}}, \quad t_{0}=n-1, \quad \delta_{0}=\delta,
$$

is finite. Hence applying that lemma to the above mentioned $\left(g, t_{0}, \delta_{0}\right)$ gives

$$
\int_{\mathbb{D} \times \mathbb{B}_{n-1}^{2}}(1-|z|)^{\delta} d d^{c}\left(\varphi_{l} \circ \tilde{F}\right)(z, \boldsymbol{\tau}) \lesssim_{\delta}\|\varphi\|_{L^{1}(V)}^{\frac{\delta}{n-1}} .
$$

On the other hand, since $d d^{c} \varphi_{l} \circ \tilde{F}$ converges weakly to $d d^{c} \varphi_{l} \circ \tilde{F}$ on $\mathbb{D}$, we have

$$
\liminf _{l \rightarrow \infty}\left\langle d d^{c}\left(\varphi_{l} \circ \tilde{F}(\cdot, \boldsymbol{\tau})\right), f\right\rangle \geq\left\langle d d^{c}(\varphi \circ \tilde{F}(\cdot, \boldsymbol{\tau})), f\right\rangle,
$$

for every positive continuous function $f$ on $\mathbb{D}$. Letting $l \rightarrow \infty$ in (4.20) and then using (4.21) and Fatou's lemma, we get the desired result.

Now we prove (4.18). As above, it is enough to prove it for $\varphi$ smooth. Set $W:=$ $\{1-2 \epsilon \leq|z| \leq 1\} \times \mathbb{B}_{n-1}^{2}$. Let $r$ be a positive constant. Denote by $W_{1}$ the subset of $W$ containing $(z, \boldsymbol{\tau})$ with $\operatorname{dist}\left(\tilde{F}(z, \boldsymbol{\tau}), K^{\prime}\right) \geq r$ and by $W_{2}$ the complement of $W_{1}$ in $W$. Let $\epsilon$ be a positive constant in $(0,1)$. Using (3.40) and the change of variables by $\tilde{F}$ on $W_{1}$ gives

$$
\int_{W_{1}}(1-|z|) d d^{c}(\varphi \circ \tilde{F}) \lesssim \epsilon \int_{W_{1}} d d^{c}(\varphi \circ \tilde{F}) \lesssim \epsilon r^{-n+1} \int_{\tilde{F}\left(W_{1}\right)} d d^{c} \varphi \wedge \omega^{n-1} \lesssim \epsilon r^{-n+1}\|\varphi\|_{L^{1}(V)} .
$$

By the proof of Lemma 4.8 applied to $g=\frac{\partial^{2} \varphi_{l}}{\partial z_{j} \partial \bar{z}_{k}}, t_{0}=n-1$ and $\delta_{0}=\delta$, we have

$$
\begin{aligned}
\int_{W_{2}}(1-|z|) d d^{c}(\varphi \circ \tilde{F}) & \leq \epsilon^{1-\delta} \int_{W_{2}}(1-|z|)^{\delta} d d^{c}(\varphi \circ \tilde{F}) \\
& \lesssim_{\delta} \epsilon^{1-\delta}\left[\int_{\tilde{F}\left(W_{2}\right)} d d^{c} \varphi \wedge \omega^{n-1}\right]^{\frac{\delta}{n-1}} \leq \epsilon^{1-\delta} r^{\delta}\|\varphi\|_{L^{1}(V)}^{\gamma}
\end{aligned}
$$

by (4.12) and the fact that $\tilde{F}\left(W_{2}\right)$ is contained in $K_{r}^{\prime} \times \mathbb{B}_{n-1}^{2}$. Choose $r:=\epsilon^{\frac{\delta}{\delta+n-1}}$. Taking the sume of the last two inequalities gives (4.18). The proof is finished.

\section{HÖLDER CONTINUITY FOR SUPER-POTENTIALS}

Recall that $\mathscr{C}$ defined at Introduction is a compact subset of the set of $\omega$-p.s.h. functions on $X$ with respect to $L^{1}$-topology. Hence there is a positive number $r_{0}$ such that

$$
\left\|\varphi_{0}\right\|_{L^{1}(X)} \leq r_{0} \text { and }\left\|\max \left\{\varphi_{1}, \varphi_{2}\right\}\right\|_{L^{1}(X)} \leq r_{0},
$$

for every $\varphi_{0}, \varphi_{1}, \varphi_{2} \in \mathscr{C}$. Let $\mathscr{C}^{\prime}$ be the set of $\omega$-p.s.h. functions $\varphi$ on $X$ such that $\|\varphi\|_{L^{1}(X)} \leq 2 r_{0}$. In this section, we will finish the proof of Theorem 1.4. In order to do so, we will prove the following which is actually equivalent to Theorem 1.4 by Lemma 5.2 below. Remember that we are still assuming that $\operatorname{dim} K=n$. Let $\tilde{K}$ be the compact subset of $K$ as in Theorem 1.4 . 
Proposition 5.1. Let $\alpha$ be a positive number strictly less than $1 /(3 n)$. Then for any $\varphi_{1}, \varphi_{2} \in$ $\mathscr{C}^{\prime}$ such that $\varphi_{1} \geq \varphi_{2}$, we have

$$
\int_{\tilde{K}}\left(\varphi_{1}-\varphi_{2}\right) d v o l_{K} \leq c \int_{X}\left(\varphi_{1}-\varphi_{2}\right) d v o l_{X}+c\left(\int_{X}\left(\varphi_{1}-\varphi_{2}\right) d v o l_{X}\right)^{\alpha},
$$

where $c$ is a constant independent of $\varphi_{1}, \varphi_{2}$.

Lemma 5.2. Proposition 5.1 implies Theorem 1.4

Proof. Take $\varphi_{1}, \varphi_{2} \in \mathscr{C}$. Observe that $\max \left\{\varphi_{1}, \varphi_{2}\right\}, \varphi_{1}, \varphi_{2} \in \mathscr{C}^{\prime}$ and $\max \left\{\varphi_{1}, \varphi_{2}\right\} \geq \varphi_{j}$ for $j=1,2$. Hence, we can apply (5.1) to $\max \left\{\varphi_{1}, \varphi_{2}\right\}, \varphi_{j}$ with $j=1,2$. Using these two inequalities and the fact that

$$
\left|\varphi_{1}-\varphi_{2}\right|=2 \max \left\{\varphi_{1}, \varphi_{2}\right\}-\varphi_{1}-\varphi_{2}
$$

gives

$$
\left\|\varphi_{1}-\varphi_{2}\right\|_{L^{1}\left(\mathbf{1}_{\tilde{K}} v o l_{K}\right)} \lesssim \max \left\{\left\|\varphi_{1}-\varphi_{2}\right\|_{L^{1}(X)},\left\|\varphi_{1}-\varphi_{2}\right\|_{L^{1}(X)}^{\alpha}\right\}
$$

which means that $\mathbf{1}_{\tilde{K}} v l_{K}$ has Hölder continuous super-potential with Hölder exponent $\alpha$. The proof is finished.

The remaining of this section is devoted to prove Proposition 5.1. By [3], it is enough to prove (5.1) for $\varphi_{1}, \varphi_{2}$ smooth. We will firstly show that for any nonnegative $\mathcal{C}^{2}$ function $v$ on $\overline{\mathbb{D}}$, the integral of $v$ over $\partial \mathbb{D}$ can be bounded by a quantity of the $L^{1}$-norm of $v$ over $\mathbb{D}$ and some Hölder norm of its Laplacian. This together with the exponent estimates in the last section are the key ingredients in the proof of Proposition 5.1. We will reuse the notations from Section 2 for $M=\overline{\mathbb{D}}$.

Lemma 5.3. Let $v$ be a nonnegative $\mathcal{C}^{2}$ functions on $\overline{\mathbb{D}}$. Let $\beta \in(1,2)$. Then we have

$$
\int_{\partial \mathbb{D}} v d \xi \lesssim_{\beta}\left\|d d^{c} v\right\|_{\tilde{\mathcal{C}}^{-\beta}(\overline{\mathbb{D}})}+\int_{\mathbb{D}} v
$$

Proof. By Riesz's representation formula, we have

$$
v(z)=\int_{-\pi}^{\pi} P\left(e^{i \theta}, z\right) v\left(e^{i \theta}\right) d \theta+\int_{\{|\eta|<1\}} \log \frac{|z-\eta|}{|1-z \bar{\eta}|} d d^{c} v,
$$

for $z \in \mathbb{D}$, where $P(\xi, z)$ is the Poisson kernel given by

$$
P(\xi, z)=(2 \pi)^{-1}\left(|\xi|^{2}-|z|^{2}\right)|\xi-z|^{-2} .
$$

This implies that

$$
\begin{aligned}
\int_{\mathbb{D}_{1 / 2}} v(z) & =\int_{-\pi}^{\pi} v\left(e^{i \theta}\right) d \theta \int_{z \in \mathbb{D}_{1 / 2}} P\left(e^{i \theta}, z\right)+ \\
& +\int_{\mathbb{D}} d d^{c} v(\eta) \int_{z \in \mathbb{D}_{1 / 2}} \log \frac{|z-\eta|}{|1-z \bar{\eta}|}
\end{aligned}
$$

Set

$$
f(\eta):=\int_{\{|z|<1 / 2\}} \log \frac{|z-\eta|}{|1-z \bar{\eta}|}=\int_{\{|z|<1 / 2\}} \log |z-\eta|-\int_{\{|z|<1 / 2\}} \log |1-z \bar{\eta}| .
$$

Observe that $f\left(e^{i \theta}\right)=0$ because

$$
\log \frac{\left|z-e^{i \theta}\right|}{\mid 1-z e^{-i \theta \mid}}=0
$$


for any $z \in \mathbb{D}$. This means that $\left.f\right|_{\partial \mathbb{D}} \equiv 0$. We claim that $f$ is indeed in $\tilde{\mathcal{C}}^{\beta}(\overline{\mathbb{D}})$ for every $\beta \in(1,2)$. Since $z \in \mathbb{D}_{1 / 2}$ and $\eta \in \mathbb{D}$, the function

$$
\int_{\mathbb{D}_{1 / 2}} \log |1-z \bar{\eta}| d x d y
$$

is smooth in $\eta \in \mathbb{D}$. Hence, we only need to take care of $\int_{z \in \mathbb{D}_{1 / 2}} \log |z-\eta|$. It is clear that

$$
\partial_{\eta} \int_{z \in \mathbb{D}_{1 / 2}} \log |z-\eta|=-\frac{1}{2} \int_{z \in \mathbb{D}_{1 / 2}} \frac{\bar{z}-\bar{\eta}}{|z-\eta|^{2}}=-\frac{1}{2} \int_{z \in \mathbb{D}_{1 / 2}} \frac{1}{z-\eta} .
$$

Let $g$ be the right-hand side of the last equation. We will show that $g \in \mathcal{C}^{\alpha}(\overline{\mathbb{D}})$ for every $\alpha \in(0,1)$. If we can do so, then $\partial_{\eta} f \in \mathcal{C}^{\alpha}(\overline{\mathbb{D}})$, using similar argument we also gets $\partial_{\bar{\eta}} f \in \mathcal{C}^{\alpha}(\overline{\mathbb{D}})$, hence $f \in \tilde{\mathcal{C}}^{\beta}(\overline{\mathbb{D}})$ for $\beta \in(1,2)$. Let $\alpha \in(0,1)$. For $\left(\eta, \eta^{\prime}\right) \in \mathbb{D}^{2}$, consider the difference

$$
\begin{aligned}
\left|\frac{1}{z-\eta}-\frac{1}{z-\eta^{\prime}}\right| & =\left|\frac{\eta-\eta^{\prime}}{(z-\eta)\left(z-\eta^{\prime}\right)}\right| \leq \frac{\left|\eta-\eta^{\prime}\right|^{\alpha}}{\left|(z-\eta)\left(z-\eta^{\prime}\right)\right|^{\alpha}}\left|\frac{1}{z-\eta}-\frac{1}{z-\eta^{\prime}}\right|^{1-\alpha} \\
& \leq\left|\eta-\eta^{\prime}\right|^{\alpha}\left[\frac{1}{\left.|z-\eta| \mid z-\eta^{\prime}\right)\left.\right|^{\alpha}}+\frac{1}{\mid z-\eta)\left.\right|^{\alpha}|z-\eta|}\right] .
\end{aligned}
$$

It is not difficult to see that the integration of the right-hand side of (5.6) over $z \in \mathbb{D}_{1 / 2}$ is bounded by $\left|\eta-\eta^{\prime}\right|^{\alpha}$ times a constant depending only on $\alpha$. Thus one gets $g \in \mathcal{C}^{\alpha}(\overline{\mathbb{D}})$. As explained above, this yields $f \in \tilde{\mathcal{C}}^{\beta}(\overline{\mathbb{D}})$. The last property combined with (5.4) gives

$$
\left|\int_{-\pi}^{\pi} v\left(e^{i \theta}\right) d \theta \int_{z \in \mathbb{D}_{1 / 2}} P\left(e^{i \theta}, z\right)\right| \leq\|v\|_{L^{1}\left(\mathbb{D}_{1 / 2}\right)}+\left\|d d^{c} v\right\|_{\tilde{\mathcal{C}}^{-\beta}(\overline{\mathbb{D}})}\|f\|_{\tilde{\mathcal{C}}^{\beta}(\overline{\mathbb{D}})} .
$$

By our hypothesis that $v \geq 0$ and the fact that $P\left(e^{i \theta}, z\right) \gtrsim 1$ for $z \in \mathbb{D}_{1 / 2}$, using (5.7), one obtains that

$$
\int_{\partial \mathbb{D}} v d \xi \lesssim \beta\|v\|_{L^{1}\left(\mathbb{D}_{1 / 2}\right)}+\left\|d d^{c} v\right\|_{\tilde{\mathcal{C}}^{-\beta}(\overline{\mathbb{D}})}
$$

The proof is finished.

Proposition 5.4. Let $v$ be a nonnegative $\mathcal{C}^{2}$ function on $\overline{\mathbb{D}}$. Let $\epsilon, \beta_{0} \in(0,1)$ and $\beta \in(1,2)$. Let $\gamma$ be the unique real number for which $\beta=\gamma \beta_{0}+(1-\gamma) 2$. Then we have

$$
\begin{aligned}
& \int_{\partial \mathbb{D}} v d \xi \lesssim\left(\beta_{0}, \beta\right)\|v\|_{L^{1}(\mathbb{D})}+\epsilon^{-2(1-\gamma)}\left\|d d^{c} v\right\|_{\tilde{\mathcal{C}}^{-\beta_{0}(\overline{\mathbb{D}})}}^{\gamma}\|v\|_{L^{1}(\mathbb{D})}^{1-\gamma}+ \\
& +\left\|d d^{c} v\right\|_{\tilde{\mathcal{C}}^{-\beta_{0}}(\overline{\mathbb{D}})}^{\gamma}\left[\int_{1-2 \epsilon \leq|z| \leq 1}(1-|z|)\left|d d^{c} v\right|\right]^{1-\gamma} .
\end{aligned}
$$

Proof. Firstly we will estimate $\left\|d d^{c} v\right\|_{\tilde{\mathcal{C}}^{-2}(\overline{\mathbb{D}})}$. Let $\chi \in \mathcal{C}^{\infty}(\mathbb{R})$ such that $0 \leq \chi \leq 1, \chi \equiv 0$ on $[-1,1]$ and $\chi \equiv 1$ outside $[-2,2]$. For $\epsilon \in(0,1)$, put $\chi_{\epsilon}(z):=\chi\left(\frac{1-|z|}{\epsilon}\right)$ for $z \in \mathbb{D}$. We have $\operatorname{supp} \chi_{\epsilon} \subset\{z:|z| \leq 1-\epsilon\}$ and $\chi_{\epsilon}(z)=1$ for $z$ with $|z| \leq 1-2 \epsilon$. Let $\Phi$ be a function in $\tilde{\mathcal{C}}^{2}(\overline{\mathbb{D}})$ with $\|\Phi\|_{\mathcal{C}^{2}} \leq 1$. Since $\Phi \equiv 0$ on $\partial \mathbb{D}$ we have $|\Phi(z)| \leq 1-|z|$. Decompose

$$
\left\langle d d^{c} v, \Phi\right\rangle=\left\langle d d^{c} v, \chi_{\epsilon} \Phi\right\rangle+\left\langle d d^{c} v,\left(1-\chi_{\epsilon}\right) \Phi\right\rangle .
$$


Denote by $I_{1}, I_{2}$ respectively the first and second terms in the right-hand side of the last equality. By properties of $\Phi$ and $\chi_{\epsilon}$, one gets

$$
\left|I_{2}\right| \leq 2 \int_{1-2 \epsilon \leq|z| \leq 1}(1-|z|)\left|d d^{c} v\right| .
$$

On the other hand, performing an integration by parts gives

$$
\left|I_{1}\right| \leq \int_{\mathbb{D}}\left|v d d^{c}\left(\chi_{\epsilon} \Phi\right)\right| \lesssim \epsilon^{-2} \int_{\mathbb{D}}|v|
$$

Hence, we obtain

$$
\left\|d d^{c} v\right\|_{\tilde{\mathcal{C}}^{-2}(\overline{\mathbb{D}})}=\sup _{\left\{\Phi \in \tilde{\mathcal{C}}^{2}(\mathbb{D}):\|\Phi\|_{\mathcal{C}^{2}} \leq 1\right\}}\left|\left\langle d d^{c} v, \Phi\right\rangle\right| \lesssim \epsilon^{-2} \int_{\mathbb{D}}|v|+\int_{1-2 \epsilon \leq|z| \leq 1}(1-|z|)\left|d d^{c} v\right| .
$$

Now applying Proposition 2.1 to $d d^{c} v$ and $M=\overline{\mathbb{D}}$, one gets

$$
\left\|d d^{c} v\right\|_{\tilde{\mathcal{C}}^{-\beta}(\overline{\mathbb{D}})} \lesssim\left\|d d^{c} v\right\|_{\tilde{\mathcal{C}}^{-\beta_{0}(\overline{\mathbb{D}})}}^{\gamma}\left\|d d^{c} v\right\|_{\tilde{\mathcal{C}}^{2}(\overline{\mathbb{D}})}^{1-\gamma} .
$$

The last inequality combined with (5.2) and (5.10) gives (5.9). The proof is finished.

We are now about to prove the local version of Proposition 5.1. Given a point $a \in K$, a small open neighborhood $K^{\prime}$ of $a$ in $K$ can be described as in Section 3. Namely, there are a $\mathcal{C}^{3}$ map $h$ from $\overline{\mathbb{B}}_{n}$ to $\mathbb{R}^{n}$ with $h(0)=D h(0)=0$ and local holomorphic coordinates in $X$ such that

$$
K^{\prime}:=\left\{\mathbf{x}+i h(\mathbf{x}): \mathbf{x} \in \mathbb{B}_{n}\right\} \subset \mathbb{D}_{2}^{n} .
$$

Let $\tilde{F}(z, \boldsymbol{\tau}), t_{3}, \tilde{\epsilon}_{0}^{\prime}$ and $\tilde{\theta}_{0}$ be as in Section 4. The couple $\left(K^{\prime}, \mathbb{D}_{2}^{n}\right)$ is considered as the local counterpart of $(K, X)$. One can replace $\mathbb{D}_{2}^{n}$ by any polydisks $\mathbb{D}_{r}^{n}$ with $r>1$ without making any differences in what follows.

Let $\beta_{0} \in(0,1)$. For every positive continuous $(1,1)$-current $T$ on an open neighborhood of $\overline{\mathbb{D}}$, we have

$$
\|T\|_{\tilde{\mathcal{C}}^{-\beta_{0}(\overline{\mathbb{D}})}} \leq \int_{\mathbb{D}}(1-|z|)^{\beta_{0}} T .
$$

Let $\varphi_{1}$ and $\varphi_{2}$ be two $\mathcal{C}^{2}$ p.s.h. functions on $\mathbb{D}_{2}^{n}$ such that $\varphi_{1} \geq \varphi_{2}$ and $\left\|\varphi_{j}\right\|_{L^{1}\left(\mathbb{D}_{2}^{n}\right)} \leq 1$ for $j=1,2$. Put $\varphi:=\varphi_{1}-\varphi_{2}$ which is $\mathcal{C}^{2}$ and nonnegative. Define

$$
g_{1}(\boldsymbol{\tau}):=\left\|d d^{c}(\varphi \circ \tilde{F}(\cdot, \boldsymbol{\tau}))\right\|_{\tilde{\mathcal{C}}^{-\beta_{0}}(\overline{\mathbb{D}})}
$$

which is less than or equal to

$$
\left\|d d^{c}\left(\varphi_{1} \circ \tilde{F}(\cdot, \boldsymbol{\tau})\right)\right\|_{\tilde{\mathcal{C}}^{-\beta_{0}}(\overline{\mathbb{D}})}+\left\|d d^{c}\left(\varphi_{2} \circ \tilde{F}(\cdot, \boldsymbol{\tau})\right)\right\|_{\tilde{\mathcal{C}}^{-\beta_{0}}(\overline{\mathbb{D}})} .
$$

Since $\tilde{F}$ is $\mathcal{C}^{2}$, so is $\varphi_{j} \circ \tilde{F}$ for $j=1,2$. Using (5.11) for $T=d d^{c}\left(\varphi_{j} \circ \tilde{F}(\cdot, \boldsymbol{\tau})\right)$ and (4.17), we deduce that the integral of the sum (5.12) with respect to $\tau \in \mathbb{B}_{n-1}^{2}$ is $\lesssim_{\beta_{0}} 1$. This implies

$$
\int_{\mathbb{B}_{n-1}^{2}} g_{1}(\boldsymbol{\tau}) d \boldsymbol{\tau} \lesssim \beta_{0} 1
$$

Put

$$
g_{2}(\boldsymbol{\tau}):=\|\varphi \circ \tilde{F}(\cdot, \boldsymbol{\tau})\|_{L^{1}(\mathbb{D})}
$$


By Corollary 4.2, the function $\varphi$ satisfy the hypothesis of Lemma 4.8 for $\delta_{0}=0$ and $t_{0}=n-1+\epsilon$ with $\epsilon \in(0,1)$. As a result, we get

$$
\int_{\mathbb{B}_{n-1}^{2}} g_{2}(\boldsymbol{\tau}) d \boldsymbol{\tau} \lesssim \epsilon\left[\int_{\mathbb{B}_{2}^{n}}|\varphi|\right]^{\frac{\epsilon}{n-1+\epsilon}} .
$$

For $\epsilon^{\prime} \in(0,1)$, we define

$$
g_{3}\left(\boldsymbol{\tau}, \epsilon^{\prime}\right):=\int_{1-2 \epsilon^{\prime} \leq|z| \leq 1}(1-|z|) d d^{c}\left(\varphi_{1} \circ \tilde{F}(\cdot, \boldsymbol{\tau})\right)+\int_{1-2 \epsilon^{\prime} \leq|z| \leq 1}(1-|z|) d d^{c}\left(\varphi_{2} \circ \tilde{F}(\cdot, \boldsymbol{\tau})\right) .
$$

By (4.18), we have

$$
\int_{\mathbb{B}_{n-1}^{2}} g_{3}\left(\boldsymbol{\tau}, \epsilon^{\prime}\right) d \boldsymbol{\tau} \lesssim \delta\left(\epsilon^{\prime}\right)^{1-\frac{\delta(n-1)}{n-1+\delta}}
$$

for any $\delta \in(0,1)$.

Proposition 5.5. Let $\varphi_{1}$ and $\varphi_{2}$ be two $\mathcal{C}^{2}$ p.s.h. functions on $\mathbb{D}_{2}^{n}$ such that $\varphi_{1} \geq \varphi_{2}$ and $\left\|\varphi_{j}\right\|_{L^{1}\left(\mathbb{D}_{2}^{n}\right)} \leq 1$ for $j=1,2$. Let $\varphi:=\varphi_{1}-\varphi_{2}$. Then we have

$$
\int_{\mathbb{B}_{n}\left(0, \epsilon_{0}^{\prime *}\right)} \varphi(\mathbf{x}, h(\mathbf{x})) d \mathbf{x} \lesssim_{\delta}\|\varphi\|_{L^{1}\left(\mathbb{D}_{2}^{n}\right)}^{\frac{1}{3 n}-\delta}
$$

for any $\delta \in\left(0, \frac{1}{3 n}\right)$.

Proof. Let $\epsilon, \epsilon^{\prime}, \beta_{0} \in(0,1)$ and $\beta \in(1,2)$. Let $g_{1}, g_{2}, g_{3}$ be as above. Applying Lemma 4.7 to $g=\varphi$ gives

$$
\int_{\mathbb{B}_{n}\left(0, \epsilon_{0}^{\prime *}\right)}|\varphi(\mathbf{x}, h(\mathbf{x}))| d \mathbf{x} \lesssim \int_{\mathbb{B}_{n-1}^{2}} d \boldsymbol{\tau} \int_{\partial \mathbb{D}}|\varphi \circ \tilde{F}(\cdot, \boldsymbol{\tau})| d \xi
$$

Put $\gamma:=\frac{2-\beta}{2-\beta_{0}}$. Applying Proposition 5.4 to $v=\varphi \circ \tilde{F}(\cdot, \boldsymbol{\tau}) \in \mathcal{C}^{2}$ shows that the right-hand side of the last inequality is

$$
\lesssim\left(\beta_{0}, \beta\right) \int_{\mathbb{B}_{n-1}^{2}} g_{2} d \boldsymbol{\tau}+\left(\epsilon^{\prime}\right)^{-2(1-\gamma)} \int_{\mathbb{B}_{n-1}^{2}} g_{1}^{\gamma} g_{2}^{1-\gamma} d \boldsymbol{\tau}+\int_{\mathbb{B}_{n-1}^{2}} g_{1}^{\gamma} g_{3}^{1-\gamma}\left(\cdot, \epsilon^{\prime}\right) d \boldsymbol{\tau} .
$$

The first term of the last sum is

$$
\lesssim \epsilon\|\varphi\|_{L^{1}\left(\mathbb{D}_{2}^{n}\right)}^{\frac{\epsilon}{n-1+\epsilon}}
$$

by (5.14). On the other hand, by the Hölder inequality, the second one is $\leq$

$$
\left(\epsilon^{\prime}\right)^{-2(1-\gamma)}\left\|g_{1}\right\|_{L^{1}}^{\gamma}\left\|g_{2}\right\|_{L^{1}}^{1-\gamma}
$$

and the third one is $\leq$

$$
\left\|g_{1}\right\|_{L^{1}}^{\gamma}\left\|g_{3}\left(\cdot, \epsilon^{\prime}\right)\right\|_{L^{1}}^{1-\gamma}
$$

where the $L^{1}$-norm is taken over $\mathbb{B}_{n-1}^{2}$. Taking into account (5.13) and (5.14), one obtains

$$
\left(\epsilon^{\prime}\right)^{-2(1-\gamma)}\left\|g_{1}\right\|_{L^{1}}^{\gamma}\left\|g_{2}\right\|_{L^{1}}^{1-\gamma} \lesssim_{\beta_{0}, \epsilon}\left(\epsilon^{\prime}\right)^{-2(1-\gamma)}\|\varphi\|_{L^{1}\left(\mathbb{D}_{2}^{n}\right)}^{\frac{\epsilon(1-\gamma)}{n-1+\epsilon}}
$$

By (5.13) and (5.15), we have

$$
\left\|g_{1}\right\|_{L^{1}}^{\gamma}\left\|g_{3}\left(\cdot, \epsilon^{\prime}\right)\right\|_{L^{1}}^{1-\gamma} \lesssim \beta_{0}, \delta\left(\epsilon^{\prime}\right)^{\left(1-\frac{\delta(n-1)}{n-1+\delta}\right)(1-\gamma)}
$$


for every $\epsilon^{\prime} \in(0,1)$. Put

$$
a_{1}:=\frac{\epsilon}{(n-1+\epsilon)\left(3-\frac{\delta(n-1)}{n-1+\delta}\right)}, \quad a_{2}:=\frac{\epsilon\left(1-\frac{\delta(n-1)}{n-1+\delta}\right)(1-\gamma)}{(n-1+\epsilon)\left(3-\frac{\delta(n-1)}{n-1+\delta}\right)} .
$$

Choose $\epsilon^{\prime}:=\|\varphi\|_{L^{1}\left(\mathbb{D}_{2}^{n}\right)}^{a_{1}}$. Combining all these above inequalities, we get

$$
\int_{\mathbb{B}_{n}\left(0, \epsilon_{0}^{\prime *}\right)}|\varphi(\mathbf{x}, h(\mathbf{x}))| d \mathbf{x} \lesssim\left(\beta_{0}, \beta, \delta, \epsilon\right)\|\varphi\|_{L^{1}\left(\mathbb{D}_{2}^{n}\right)}^{a_{2}} .
$$

Observe that $a_{2} \rightarrow \frac{1}{3 n}$ as $\epsilon \rightarrow 1, \beta \rightarrow 2, \beta_{0} \rightarrow 0, \delta \rightarrow 0$. Thus, the proof is finished.

End of the proof of Proposition 5.1 in the case where $\operatorname{dim} K=n$. Given any $a \in K$, let $\tilde{F}_{a}$ and $\tilde{\epsilon}_{a}$ be as in Proposition 3.8. Since $\tilde{K}$ is compact, we can cover it by a finite number of ball $B_{K}\left(a, \tilde{\epsilon}_{a}\right)$. Hence, in order to prove (5.1), it is enough to restrict ourselves to local charts. In other words, we are now being in the situation with the model $\left(K^{\prime}, \mathbb{D}_{2}^{n}\right)$ described above. Moreover, by subtracting a suitable common smooth function, we can assume that $\varphi_{1}, \varphi_{2}$ in (5.1) are $\mathcal{C}^{2}$ p.s.h. functions on $\mathbb{D}_{2}^{n}$. Hence, the desired result follows directly from Proposition 5.5, The proof is finished.

We now deal with the case where the dimension of $K$ is greater than $n$. Let $n_{K}:=$ $\operatorname{dim} K>n$. Since $K$ is generic, we have $T_{a} K+J T_{a} K=T_{a} X$, where $a \in K$ and $J$ is the complex structure of $X$. We then deduce that $T_{a} K \cap J T_{a} K$ is of even dimension which equals $2 n_{K}-2 n$. The codimension $d$ of $K$ equals $2 n-n_{K}$.

Proposition 5.6. Let a be a point in $K$. There exist local $\mathcal{C}^{2}$ coordinates $(W, \Psi)$ of $X$ around a such that the following properties hold:

(i) $\Psi: W \rightarrow \mathbb{C}^{d} \times \mathbb{C}^{n_{K}-n}$ is a $\mathcal{C}^{2}$ diffeomorphism onto its image which equals

$$
\left(\mathbb{B}_{d}+i \mathbb{B}_{d}(0,2)\right) \times \mathbb{D}^{n_{K}-n}
$$

and $\Psi(p)=0$ and $\Psi^{-1}\left(\mathbf{z}_{1}, \mathbf{z}_{2}\right)$ is holomorphic in $\mathbf{z}_{1}$ for every fixed $\mathbf{z}_{2} \in \mathbb{D}^{n_{K}-n}$,

(ii) there is a $\mathcal{C}^{2}$ map $h\left(\operatorname{Re} \mathbf{z}_{1}, \mathbf{z}_{2}\right)$ from $\overline{\mathbb{B}}_{d} \times \mathbb{D}^{n_{K}-n}$ to $\mathbb{R}^{d}$ so that for every $\mathbf{z}_{2}$ fixed, $h\left(\cdot, \mathbf{z}_{2}\right) \in \mathcal{C}^{3}$ and

$$
D_{\operatorname{Re} \mathbf{z}_{1}}^{j} h\left(0, \mathbf{z}_{2}\right)=0
$$

for $j=0$ or 1 and

$$
\Psi(K \cap W)=\left\{\left(\mathbf{z}_{1}, \mathbf{z}_{2}\right) \in\left(\mathbb{B}_{d}+i \mathbb{R}^{d}\right) \times \mathbb{D}^{n_{K}-n}: \operatorname{Im} \mathbf{z}_{1}=h\left(\operatorname{Re} \mathbf{z}_{1}, \mathbf{z}_{2}\right)\right\} .
$$

Proof. It is well-known that in suitable holomorphic local coordinates, $K$ is given by

$$
K=\left\{\left(\mathbf{z}_{1}, \mathbf{z}_{2}\right) \in\left(\mathbb{B}_{d}+i \mathbb{R}^{d}\right) \times \mathbb{D}^{n_{K}-n}: \operatorname{Im} \mathbf{z}_{1}=\tilde{h}\left(\operatorname{Re} \mathbf{z}_{1}, \operatorname{Re} \mathbf{z}_{2}, \operatorname{Im} \mathbf{z}_{2}\right)\right\}
$$

where $\tilde{h}$ is a $\mathcal{C}^{3}$ map of uniformly bounded $\mathcal{C}^{3}$ norm in $p$ and $\tilde{h}(0)=D \tilde{h}(0)=0$, see [1] . For $\mathbf{z}_{2}$ fixed, we choose the tangent space of the graph of $\tilde{h}\left(\cdot, \mathbf{z}_{2}\right)$ at 0 and its orthogonal subspace as new holomorphic coordinates of $\mathbb{C}^{d}$. These new coordinates depend $\mathcal{C}^{2}$ on (but in general not holomorphically) on the parameter $\mathbf{z}_{2}$. In these new coordinates, one easily see that $K$ is given by the formula given in the asssertion (ii) for some $\mathcal{C}^{2}$ map $h$ with the desired properties. The proof is finished.

Remark 5.7. As in Lemma 3.1 we can obtain furthermore that $D_{\operatorname{Re} \mathbf{z}_{1}}^{2} h\left(0, \mathbf{z}_{2}\right)=0$ and $\left\|h\left(\cdot, \mathbf{z}_{2}\right)\right\|_{\mathcal{C}^{3}}$ is bounded uniformly in $a=\left(\mathbf{z}_{1}, \mathbf{z}_{2}\right) \in \tilde{K}$ but in this case we will lose a unit for the regularity in $\mathbf{z}_{2}$, i.e $\Psi$ and $h$ are only $\mathcal{C}^{1}$ in $\mathbf{z}_{2}$. 
Thanks to Proposition 5.6, we can consider $K$ locally as a family of generic submanifolds of $\mathbb{C}^{d}$ of dimension $d$ parameterized by $\mathbf{z}_{2} \in \mathbb{D}^{n_{K}-n}$. This allows us to reduce the question to the previous case where we already dealt with generic submanifolds of minimal dimension. By compactness of $\tilde{K}$, we can cover it by local charts $W$ as in Proposition 5.6. From now on, we work exclusively on a such local chart. Hence, we can identify $K$ with $\Psi(K \cap W)$. Let $h$ and $\Psi$ be as in that proposition. The map $h$ will be seen as a family of maps of $\mathbf{z}_{1}$ parameterized by $\mathbf{z}_{2}$. For $\mathbf{z}_{2} \in \mathbb{D}^{n_{K}-n}$, define

$$
K_{\mathbf{z}_{2}}^{\prime}:=\left\{\mathbf{z}_{1} \in\left(\mathbb{B}_{d}+i \mathbb{R}^{d}\right): \operatorname{Im} \mathbf{z}_{1}=h\left(\operatorname{Re} \mathbf{z}_{1}, \mathbf{z}_{2}\right)\right\}
$$

which is identified with $K_{\mathbf{z}_{2}}^{\prime} \times\left\{\mathbf{z}_{2}\right\} \subset \mathbb{C}^{n}$. Then $K$ is foliated by $K_{\mathbf{z}_{2}}^{\prime}$.

We are now going to construct a family of analytic discs partly attached to $K$. The strategy will be almost identical with what we did. Let $u_{0}$ be a function described in Lemma 3.2 and $\theta_{u_{0}}$ be the constant there. Let $\boldsymbol{\tau}_{1}, \boldsymbol{\tau}_{2} \in \overline{\mathbb{B}}_{d-1} \subset \mathbb{R}^{d-1}$. Define $\boldsymbol{\tau}_{1}^{*}:=$ $\left(1, \boldsymbol{\tau}_{1}\right) \in \mathbb{R}^{d}$ and $\boldsymbol{\tau}_{2}^{*}:=\left(0, \boldsymbol{\tau}_{1}\right) \in \mathbb{R}^{d}$ and $\boldsymbol{\tau}:=\left(\boldsymbol{\tau}_{1}, \boldsymbol{\tau}_{2}\right)$. Let $t$ be a positive number in $(0,1)$. Consider the following modified version of the equation 3.11:

$$
U_{\boldsymbol{\tau}, \mathbf{z}_{2}, t}(\xi)=t \boldsymbol{\tau}_{2}^{*}-\mathcal{T}_{1}\left(h\left(U_{\boldsymbol{\tau}, \mathbf{z}_{2}, t} ; \mathbf{z}_{2}\right)\right)(\xi)-t \mathcal{T}_{1} u_{0}(\xi) \boldsymbol{\tau}_{1}^{*}
$$

where $U: \partial \mathbb{D} \rightarrow \mathbb{B}_{d}$ is Hölder continuous.

Since $h\left(0, \mathbf{z}_{2}\right)=D_{\operatorname{Re} \mathbf{z}_{1}} h\left(0, \mathbf{z}_{2}\right)=0$ for every $\mathbf{z}_{2}$, we can use the same reason mentioned in the proof of Proposition 3.3 to show that if $t$ is small enough, the equation (5.17) has a unique solution $U_{\boldsymbol{\tau}, \mathbf{z}_{2}, t}$ in $\mathcal{C}^{2,1 / 2}\left(\partial \mathbb{D} \times \mathbb{B}_{d-1}^{2}\right)$ for $\mathbf{z}_{2}$ fixed so that $U_{\boldsymbol{\tau}, \mathbf{z}_{2}, t} \in \mathcal{C}^{1}$ as a function of $\left(z, \boldsymbol{\tau}, \mathbf{z}_{2}\right)$. We use the same notation $U_{\boldsymbol{\tau}, \mathbf{z}_{2}, t}$ to denote the harmonic extension of $U_{\boldsymbol{\tau}, \mathbf{z}_{2}, t}$ to $\mathbb{D}$. Let $P_{\boldsymbol{\tau}, \mathbf{z}_{2}, t}(z)$ be the harmonic extension of $h\left(U_{\boldsymbol{\tau}, \mathbf{z}_{2}, t}(\xi), \mathbf{z}_{2}\right)$ to $\mathbb{D}$. Define

$$
F\left(z, \boldsymbol{\tau}, \mathbf{z}_{2}, t\right):=U_{\boldsymbol{\tau}, \mathbf{z}_{2}, t}(z)+i P_{\boldsymbol{\tau}, \mathbf{z}_{2}, t}(z)+i t u_{0}(z) \boldsymbol{\tau}_{1}^{*}
$$

which is a family of analytic discs to $\mathbb{C}^{d}$ parametrized by $\left(\boldsymbol{\tau}, \mathbf{z}_{2}, t\right)$. By our choice of $u_{0}$, we have $F\left(\xi, \boldsymbol{\tau}, \mathbf{z}_{2}, t\right) \in K_{\mathbf{z}_{2}}$ for $\xi \in\left[e^{-i \theta_{u_{0}}}, e^{i \theta_{u_{0}}}\right]$. Now define

$$
F^{\prime}\left(z, \boldsymbol{\tau}, \mathbf{z}_{2}, t\right):=\left(F_{\boldsymbol{\tau}, \mathbf{z}_{2}, t}(z), \mathbf{z}_{2}\right) \in \mathbb{C}^{n}
$$

which is a family of analytic discs to $X$ partly attached to $K$. Here we used an essential fact that the $\mathcal{C}^{2}$ coordinates $\left(\mathbf{z}_{1}, \mathbf{z}_{2}\right)$ are holomorphic in $\mathbf{z}_{1}$. Proposition 3.6 with $n$ replaced by $d$ implies that for two positive constants $\left(t, r_{0}\right)$ small enough, $F^{\prime}$ is a diffeomorphism on

$$
\left(\mathbb{B}_{2}\left(1, r_{0}\right) \cap \mathbb{D}\right) \times \overline{\mathbb{B}}_{d-1}^{2} \times \mathbb{D}^{n_{K}-n}
$$

and its differential satisfies

$$
\left|\operatorname{det} D F^{\prime}\left(z, \boldsymbol{\tau}, \mathbf{z}_{2}, t\right)\right| \gtrsim t^{d+1} \operatorname{dist}^{d-1}\left(F^{\prime}\left(z, \boldsymbol{\tau}, \mathbf{z}_{2}, t\right), K_{\mathbf{z}_{2}}^{\prime}\right) \gtrsim t^{2 d}(1-|z|)^{d-1} .
$$

Now applying the same arguments right before Proposition 3.7, one gets the following.

Proposition 5.8. There exists a map $\tilde{F}: \mathbb{D} \times \mathbb{B}_{d-1}^{2} \times \mathbb{D}^{n_{K}-n} \rightarrow X$ which is a diffeomorphism onto its image such that the following three properties hold:

(i) there are positive constants $\tilde{\theta}_{0}$ and $\tilde{\epsilon}_{0}$ so that for every $\tau_{1} \in \overline{\mathbb{B}}_{d-1}$ the restriction map $\tilde{F}\left(\cdot, \boldsymbol{\tau}_{1}\right):\left[e^{-i \tilde{\theta}_{0}}, e^{i \tilde{\theta}_{0}}\right] \times \overline{\mathbb{B}}_{d-1} \times \mathbb{D}^{n_{K}-n} \rightarrow K$ is a diffeomorphism onto its image which contains the graph of $h$ over $\mathbb{B}_{d}\left(0, \tilde{\epsilon}_{0}\right) \times \mathbb{D}^{n_{K}-n}$,

(ii) $\tilde{F}\left(\cdot, \boldsymbol{\tau}, \mathbf{z}_{2}\right)$ is an analytic disc to $X$ and

$$
\left|\operatorname{det} D \tilde{F}\left(z, \boldsymbol{\tau}, \mathbf{z}_{2}\right)\right| \gtrsim \operatorname{dist}^{d-1}\left(\tilde{F}\left(z, \boldsymbol{\tau}, \mathbf{z}_{2}, t\right), K_{\mathbf{z}_{2}}^{\prime}\right) \gtrsim(1-|z|)^{d-1} \text {. }
$$


Proposition 5.8 and Remark 5.7 allow us to repeat all of arguments in the proof of Theorem 1.4 in the case where $n_{K}=n$ for our present situation. Hence, this finishes the proof of Theorem 1.4 .

\section{REFERENCES}

[1] M. S. BaOuendi, P. Ebenfelt, AND L. P. Rothschild, Real submanifolds in complex space and their mappings, vol. 47 of Princeton Mathematical Series, Princeton University Press, Princeton, NJ, 1999.

[2] S. BELL, Mapping problems in complex analysis and the $\bar{\partial}$-problem, Bull. Amer. Math. Soc. (N.S.), 22 (1990).

[3] Z. BŁocki AND S. KoŁodzieJ, On regularization of plurisubharmonic functions on manifolds, Proc. Amer. Math. Soc., 135 (2007), pp. 2089-2093 (electronic).

[4] J.-P. DEMAilly, Regularization of closed positive currents of type $(1,1)$ by the flow of a Chern connection, in Contributions to complex analysis and analytic geometry, Aspects Math., E26, Vieweg, Braunschweig, 1994, pp. 105-126.

[5] J.-P. Demailly, S. Dinew, V. Guedj, H. H. Pham, S. KoŁodZieJ, And A. Zeriahi, Hölder continuous solutions to Monge-Ampère equations, J. Eur. Math. Soc. (JEMS), 16 (2014), pp. 619-647.

[6] S. Dinew, Uniqueness in $\mathcal{E}(X, \omega)$, J. Funct. Anal., 256 (2009).

[7] S. Dinew AND Z. Zhang, On stability and continuity of bounded solutions of degenerate complex Monge-Ampère equations over compact Kähler manifolds, Adv. Math., 225 (2010).

[8] T.-C. DinH AND V.-A. NGuYÊN, Characterization of Monge-Ampère measures with Hölder continuous potentials, J. Funct. Anal., 266 (2014), pp. 67-84.

[9] T.-C. Dinh, V.-A. NGUYÊN, AND N. SiBONY, Exponential estimates for plurisubharmonic functions and stochastic dynamics, J. Differential Geom., 84 (2010).

[10] T.-C. DinH AND N. SibONY, Super-potentials of positive closed currents, intersection theory and dynamics, Acta Math., 203 (2009), pp. 1-82.

[11] P. H. HIEP, Hölder continuity of solutions to the Monge-Ampère equations on compact Kähler manifolds, Ann. Inst. Fourier (Grenoble), 60 (2010).

[12] L. KAUfMANN, A Skoda-type integrability theorem for singular Monge-Ampère measures, to appear in Michigan Math. J.

[13] S. Ko£OdZieJ, The complex Monge-Ampère equation, Acta Math., 180 (1998), pp. 69-117.

[14] _ - The complex Monge-Ampère equation and pluripotential theory, Mem. Amer. Math. Soc., 178 (2005).

[15] _ Hölder continuity of solutions to the complex Monge-Ampère equation with the right-hand side in $L^{p}$ : the case of compact Kähler manifolds, Math. Ann., 342 (2008).

[16] S. G. KRANTZ, Geometric function theory, Cornerstones, Birkhäuser Boston, Inc., Boston, MA, 2006. Explorations in complex analysis.

[17] A. LUNARDI, Interpolation theory.http://prmat.math.unipr.it/ lunardi/LectureNotes/SNS1999.pdf.

[18] J. Merker AND E. Porten, Characteristic foliations on maximally real submanifolds of $\mathbb{C}^{n}$ and removable singularities for CR functions, IMRP Int. Math. Res. Pap., (2006), pp. 1-131.

[19] — Holomorphic extension of CR functions, envelopes of holomorphy, and removable singularities, IMRS Int. Math. Res. Surv., (2006), pp. 1-287.

[20] D. H. Phong, J. Song, AND J. StURM, Complex Monge-Ampère equations, in Surveys in differential geometry. Vol. XVII, vol. 17 of Surv. Differ. Geom., Int. Press, Boston, MA, 2012, pp. 327-410.

[21] N. Sibony, Quelques problèmes de prolongement de courants en analyse complexe, Duke Math. J., 52 (1985), pp. 157-197.

[22] H. SKодA, Sous-ensembles analytiques d'ordre fini ou infini dans $\mathbf{C}^{n}$, Bull. Soc. Math. France, 100 (1972), pp. 353-408.

[23] H. TRIEBEL, Interpolation theory, function spaces, differential operators, Johann Ambrosius Barth, Heidelberg, second ed., 1995.

[24] D.-V. Vu, Equidistribution rate for Fekete points on some real manifolds. arxiv:1512.08262, 2015.

[25] S. T. YAU, On the Ricci curvature of a compact Kähler manifold and the complex Monge-Ampère equation. I, Comm. Pure Appl. Math., 31 (1978). 
UPMC Univ Paris 06, UMR 7586, Institut de Mathématiques De Jussieu-Paris Rive Gauche, 4 PLace Jussieu, F-75005 Paris, France.

E-mail address: duc-viet.vu@imj-prg.fr 